\title{
The Structure of Hydromagnetic Shodk Waves
}

\author{
I. Non linear hydromagnetic waves in a cold plasma
}

\author{
By L. Davis *, R. Lüst, and A. Schlüter
}

Aus dem Max-Planck-Institut für Physik, Göttingen

(Z. Naturforschg. 13 a, 916-936 [1958] ; eingegangen am 13. Juni 1958)

\begin{abstract}
Es werden Wellen in einem quasi-neutralen Gas untersucht, das Ionen und Elektronen enthält und genügend kalt ist, so daß die thermischen Bewegungen vernachlässigt werden können. Außerdem werden Zusammenstöße zwischen den Teilchen vernachlässigt, jedoch nicht die Trägheitseffekte des elektrischen Stromes. Die nicht-linearen Gleichungen für unendlich ausgedehnte, ebene Kompressionswellen, die sich senkrecht zu einem homogenen Magnetfeld mit ungeänderter Form und Geschwindigkeit ausbreiten, werden auf eine einzige Diff.-Gl. 2. Ordnung für das Magnetfeld (oder für die Verschiebung der Teilchen senkrecht zur Ausbreitungsrichtung) zurückgeführt, vorausgesetzt, daß dif Teilchenbahnen keine Schleifen bilden. Diese Diff.-Gl. wird exakt durch elliptische Funktionen gelöst. Verschiedene Kurven, die durch numerische Integration erhalten wurden, beschreiben die wesentlichen Eigenschaften der Lösungen. Außerdem werden Potenzreihen-Entwicklungen in der Amplitude $a$ angegeben, wobei der Fehler in der Ordnung $a^{4}$ ist. Die Lösungen sind entweder periodische Wellenzüge oder Einzelwellen. Alle Lösungen sind symmetrisch um die Maxima und Minima der magnetischen Feldstärke. Die Wellenlängen sind von der Größenordnung des Gyrationsradius. Die Geschwindigkeiten erstreckten sich vom Wert Null bis zum doppelten der ALFvÉN-Geschwindigkeit.

Es wird die Beziehung zwischen diesen Wellen und hydromagnetischen Stoßwellen im Plasma geringer, aber nicht verschwindender Häufigkeit gaskinetischer Stöße betrachtet und es wird der Schluß gezogen, daß die gesamte Stoßfront eine Dichte hat, die durch das Produkt aus der mittleren Zeit zwischen zwei gaskinetischen Stößen und der Gasgeschwindigkeit relativ zur Stoßfront bestimmt ist. Dieses Gebiet wird ausgefüllt durch Wellenzüge, deren Struktur von der Größenordnung des Gyrationsradius ist. Zwei Analoga zu den Rankine-Hugeniot-Bedingungen werden angegeben: Eine, bei der auf beiden Seiten der Stoßfront ein Plasma der Temperatur Null mit verschiedenen Wellenzügen vorliegt; die anderen sind die üblichen magnetohydrodynamischen Stoßbedingungen, nur daß hier Bewegungen ausschließlich senkrecht zum Magnetfeld betrachtet werden. In beiden Fällen sind alle Erhaltungssätze durch die Stoßfront hindurch erfüllt, aber es scheint kein zufriedenstellender Mechanismus für die Prozesse innerhalb der Stoßfront zu existieren.
\end{abstract}

The waves in a cold (no thermal motions) quasi-neutral gas consisting of ions and electrons are treated neglecting collisions but not neglecting the inertial effects associated with the electric current. Provided the particle trajectories do not make loops, the non-linear equations for infinite plane compressional waves traveling perpendicular to a uniform magnetic field with unchanging form and speed are reduced to a single second order ordinary differential equation in the field strength (or the particle displacement normal to the direction of propagation) and solved exactly. The solutions can be expressed in terms of elliptic functions. Curves derived from numerical integration by means of a digital computer give the important properties of the waves. Power series solutions in the amplitude, $a$, are given, the error being of the order of $a^{4}$. Both wave trains and solitary waves are found, all solutions being symmetrical about maxima and minima in the field strength. The wavelengths are of the order of the gyro-radius. The velocities range from zero to twice the ALfvÉN velocity, higher velocities leading to looped trajectories. By reduction to dimensionless form, all waves are included in a two parameter family.

The relation of these waves to hydromagnetic shock waves in low density plasmas is considered and it is concluded that the entire shock will have a thickness determined by the product of the mean time between collisions and the gas velocity with respect to the shock front. This region will be occupied by wave trains whose structure is of the order of the gyro-radius. Two analogues of the RANKINEHugenior conditions are given, one in which the regions on the two sides of the "shock-front" are occupied by cold plasmas containing different wave trains, the other being the familiar hydromagnetic shock conditions except that only particle motions normal to the magnetic field are considered. In each all conservation laws are satisfied across the "shock-front" but there seems to be no satisfactory mechanism for the processes within the front.

The structure of hydromagnetic shock waves, and particularly the question as to whether the dimensions of the shock are comparable with the gyroradius or with the mean free path, is likely to be important in some of the current experiments that involve rapidly changing magnetic fields in plasmas. The thickness of such shocks is also of critical im- portance in most theories of the acceleration of cosmic rays ${ }^{1}$. For the gyro-radius of the cosmic rays is very large compared to that of the thermal ions but, except for cosmic rays of the highest energies, it

\footnotetext{
* On leave from California Institute of Technology, Pasadena, California.

1 E. Fermi, Astrophys. J. 119, 1 [1954].
} 
is small compared to the mean free path $\left(10^{14}\right.$ $\left.10^{18} \mathrm{~cm}\right)$ of the thermal particles. The shocks can only scatter cosmic rays from one helical trajectory to another, and thus contribute to the cosmic ray isotropy, if they have a structure small compared to the cosmic ray gyro-radius. However, previous studies of the structure of hydromagnetic shocks do not apply well to the interstellar gas where the collision frequency is very low compared to the gyro-frequency. This has the result that the gas pressure will not remain isotropic when the magnetic field strength changes since, at the very least, the pressure will have to be divided into a part perpendicular to the magnetic field and a part parallel to the field. The parallel part will remain proportional to the density, except for collisions, while the perpendicular part will increase faster than the density when the field strength increases.

In an attempt to see whether hydromagnetic shocks could be expected to have a structure of the order of the gyro-radius, we have investigated a particularly simple case. We consider infinite plane compressional waves traveling perpendicular to a magnetic field that is uniform except for disturbances produced by the wave. An important restric. tion is that only those waves are considered which propagate with unchanging form and speed. We assume that the gas is a completely ionized plasma and that collisions can be neglected. We assume that the temperature is so low that thermal motions are negligible. It is then easy to write the equations of motion of the individual particles in a frame of reference that moves with the wave. In this frame of reference it is possible to solve the resulting nonlinear equations exactly, without linearizing, for a considerable range of wave velocities and amplitudes. What is found is a variety of hydromagnetic waves, including both wave trains and solitary waves, that can have wavelengths of the order of the gyro-radius. These waves provide an example of a motion in a perfectly conducting medium in which the magnetic field is not proportional to the density, i.e. the matter does not slide beads on the lines of force but deviates by distances of the order of the gyro-radius. These solutions are derived and discussed in sections 1 to 9 . It is not possible, at least without additional considerations, to describe completely the structure of a hydromagnetic shock in terms of these waves. As discussed in section 10, it is clear that in part the structure of a hydromagne- tic shock in such a plasma will contain elements whose size is of the order of the gyro-radius, but that the entire shock is likely to have a thickness determined by the mean free path of the particles.

The most direct way to characterize these hydromagnetic wave trains and solitary waves and the medium in which they propagate is in terms of the average density of particles, the average strength of the magnetic field, the frequency, and the amplitude of the waves. The analysis is often simplified if the frequency is replaced by the velocity of the waves through the medium. Alternatively one may characterize both the waves and the medium through which they travel by certain integrals of the motion, such as the particle, momentum, and energy flux densities and the transverse electric field observed in a coordinate system that moves with the waves. It is convenient to designate these as the "mean density" and the "flux" characterizations, respectively. The flux characterization is very convenient for the derivation and analysis of the equations of motion. It facilitates the conversion of the atomic treatment into a macroscopic treatment based on similar integrals of the motion, and it connects easily with discussions of shock waves, which are usually based on such integrals. In any further development of the problem, the fluxes should play a fundamental role and this method of specification of the system should remain the most basic. Nevertheless, the mean density characterization is more natural and more useful in some circumstances, particularly when we wish to think of waves propagated through a stationary medium. For then the fluxes seem somewhat artificial while the use of mean densities separates the properties of the medium from those of the waves. Accordingly, although the problem is first treated in terms of the fluxes, convenient formulas are then developed that enable one to convert from either mode of description to the other.

\section{Atomic equations of motion for a cold plasma}

Consider an infinite train of plane compressional to a magnetic field that is uniform except for the disturbance by the waves. Use an inertial frame of reference moving with the waves. The unchanging form and speed of the waves means that in this coordinate system nothing varies with time. Orient the $x$-axis in the direction of propagation through the 
plasma and the $y$-axis along the magnetic field. Then all quantities vary only with $x$. By compressional waves it is meant that the density varies with $x$ and that they do not resemble the transverse Alfvén waves. The restoring force that produces the wave motions is the gradient of the anisotropic stress tensor associated with the magnetic field. The plasma is one in which the particles are either ions of a single kind, of charge $q_{\mathrm{i}}=e$ and mass $m_{\mathrm{i}}$, or electrons of charge $q_{\mathrm{e}}=-e$ and mass $m_{\mathrm{e}}$. By a cold plasma it is meant that all ions in any particular plane $x=$ const have the same velocity, $v_{\mathrm{i}}(x)$, and all the electrons have the velocity $v_{\mathrm{e}}(x)$, there being no thermal motion. Gaussian units will be used for all electromagnetic quantities.

Suppose the magnetic field to be uniform except for the disturbance produced by the waves and of the form

$$
\boldsymbol{B}(x)=B(x) \boldsymbol{e}_{y}
$$

where $\boldsymbol{e}_{x}, \boldsymbol{e}_{y}, \boldsymbol{e}_{z}$ are the usual unit vectors. There will be an induced electric field of the form

$$
\boldsymbol{E}(x)=E_{x}(x) \boldsymbol{e}_{x}+E_{z} \boldsymbol{e}_{z}=E_{x}(x) \boldsymbol{e}_{x}-E \boldsymbol{e}_{z} .
$$

In the steady state curl $\boldsymbol{E}=0$ and hence $E_{z}$ must be a constant which we denote by $-E$ so that $E$ will be greater than zero for positive $B$ and $u$. It is one of the principal integrals of the motion. The particle density is $n_{p}(x)$, where the subscript $p$ may be either $\mathrm{i}$ or $\mathrm{e}$. The particle velocities and their components will be denoted by

$$
\boldsymbol{v}_{p}(x)=u_{p}(x) \boldsymbol{e}_{x}+w_{p}(x) \boldsymbol{e}_{z}
$$

where velocities in the direction of the magnetic field are zero because the plasma is cold and because no forces act in the $y$-direction. If desired, the particles could be given any distribution of nonrelativistic $y$-velocities without changing any other property of the solution.

It seems convenient first to state the basic equations of motion and then to discuss their derivations and interpretation. They are:

$$
\begin{gathered}
n_{\mathrm{i}}(x)=n_{\mathrm{e}}(x)=N(x) \\
n_{p}(x) u_{p}(x)=F_{p}^{*}, \quad(p=\mathrm{i}, \mathrm{e}) \\
\frac{\mathrm{d} B}{\mathrm{~d} x}=4 \pi j_{z} / c=4 \pi(e / c) N(x)\left[w_{\mathrm{i}}(x)-w_{\mathrm{e}}(x)\right] \\
m_{p} \dot{u}_{p}=m_{p} u_{p} \frac{\mathrm{d} u_{p}}{\mathrm{~d} x}=q_{p}\left[E_{x}-B\left(w_{p} / c\right)\right] \\
m_{p} \dot{w}_{p}=m_{p} u_{p} \frac{\mathrm{d} w_{p}}{\mathrm{~d} x}=q_{p}\left[-E+B\left(u_{p} / c\right)\right]
\end{gathered}
$$

Equation (4) defines $N(x)$ and states that the plasma is everywhere electrically neutral, the $x$-component of the electric field ultimately being determined by (7). This is, of course, an approximation; but under most circumstances of interest the ratio of the Debye shielding length to the gyro-radius for electrons, $0.12 B n_{\mathrm{e}}^{-1 / 2}$ (cgs units), is very small compared to unity and hence the approximation is a good one. Since we do not impose the condition $\operatorname{div} \boldsymbol{E}=0$, the (quasi-) neutrality of the plasma does not preclude the presence of the very small space charges necessary to produce $\boldsymbol{E}$. If one did not make this approximation, each $w_{p}$ in (6) would have to be multiplied by its own $n_{p}$ and one would have to replace (4) by $\operatorname{div} \boldsymbol{E}=4 \pi e\left(n_{\mathrm{i}}-n_{\mathrm{e}}\right)$. The solution would contain the usual plasma oscillations slightly modified by the magnetic field and hydromagnetic waves slightly modified by deviations from electrical neutrality. It is convenient to speak of $N$ as the particle density, but of course it is really the density of electron-ion pairs.

Equation (5) is the statement of the conservation of particles, $F_{p}{ }^{*}$ being the constant flux of particles of kind $p$ in the $x$-direction.

Equation (6) is the direct expression of curl B $=4 \pi \boldsymbol{j} / c$ when $\boldsymbol{j}$ is expressed in terms of particle velocities and densities. The displacement current is zero in the coordinate system used, (its neglect in any other coordinate system would be consistent with our neglect of charge separation).

Equations (7) and (8) are then equations of motion of a charged particle in electromagnetic fields. From the atomic point of view it seems most natural to denote the components of the acceleration by $\dot{u}_{p}$ and $\dot{w}_{p}$, where the dot means a derivative with respect to time of a function that refers to a particular particle. If one regards the medium as a fluid, the second method of expression in terms of substantial derivative is preferable. Here all quantities are functions of position in space and vary only with $x$; hence partial derivatives are not used. Even here the use of the dot as an abbreviation for the substantial derivative is very convenient and greatly facilitates the analysis. For a cold plasma the two formulations are completely equivalent; if the temperature is not zero only the former can be used if one wishes to treat the motions of the individual particles and only the latter if one wishes to treat each kind of particle as a fluid. 
In (5) and (6) it is assumed that for each plane $x=$ const all particles of a given species will have the same velocity. Not only does this restrict us to a cold gas in which there are no thermal motions, it also requires that the particle trajectories do not make loops. In other words, $z_{p}$ is a singlevalued function of $x$ along a trajectory and $u_{p}(x)$ is never negative.

If the trajectories do make loops, the equations must be suitably modified and become much more complicated. In particular, the simple connection found below in (18) between $B$ and the displacement of the particles in the $z$-direction is no longer valid since at each point of a loop there are three values of $z$ and only one of $B$. Also the $n_{p}(x)$ of (5) refers to only one branch of the loop while the $n_{p}(x)$ in (4) is the sum of the densities on all branches. Although the presence of loops thus complicates the equations and makes them more numerous, they could be integrated numerically with a fast machine if one wished. One should probably start at the value of $x$ where on branch for each kind of particle has its maximum deflection and the other two branches cross in a symmetrical way. It would be necessary to guess the starting $z_{p}$ values and slopes at the crossing points and the $z_{\theta}$ value for the electron maximum in such a way that when the integration was completed, the branches would join smoothly. This would require using a number of successively refined trial starting values. Unless the contrary is explicitly stated, the present discussion is restricted to those cases where the trajectories do not make loops and hence the parameters characterizing a wave must be suitably limited in range.

\section{Integrals of the motion}

In (5) a different flux was allowed for ions and electrons in case the waves introduce a small drift of the electrons through the ions. However, if we divide (8) by $u_{p}$, sum over $p$, and integrate over a very long distance $\Delta$, we get, with the aid of (4) and (5),

$$
\frac{1}{\Delta}\left[m_{\mathrm{i}} w_{\mathrm{i}}+m_{\mathrm{e}} w_{\mathrm{e}}\right]_{x_{1}}^{x_{1}+\Delta}=e E\langle N\rangle\left(\frac{1}{F_{\mathrm{e}}{ }^{*}}-\frac{1}{F_{\mathrm{i}}^{*}}\right)
$$

where $\langle N\rangle$ is the average value of $N(x)$. If the quantity in square brackets is not to grow without limit as $\Delta \rightarrow \infty$, we must have $F_{\mathrm{i}}{ }^{*}=F_{\mathrm{e}}{ }^{*}$. It then follows that the quantity in square brackets is independent of $x$, and if the velocity in the $z$-direction of our inertial frame of reference is properly chosen

$$
m_{\mathrm{i}} w_{\mathrm{i}}+m_{\mathrm{e}} \boldsymbol{w}_{\mathrm{e}}=0 .
$$

With $F_{\mathrm{i}}{ }^{*}=F_{\mathrm{e}}{ }^{*}$ it follows from (4) and (5) that both kinds of particles have the same velocity,
$U(x)$, in the $x$-direction, and we have

$$
u_{\mathrm{i}}(x)=u_{\mathrm{e}}(x)=U(x)=F / m_{\mathrm{t}} N(x), \quad m_{\mathrm{t}}=m_{\mathrm{i}}+m_{\mathrm{e}}
$$

where $F$, the mass flow, joins $E$ as one of our principal integrals of the motion and $m_{\mathrm{t}}$ is the total mass of an electron-ion pair. The exclusion of looped trajectories is ensured by requiring that

$$
U(x) \geqq 0 \text {. }
$$

If we sum (7) over $p$ and use (10) and (6) we get

$$
F \frac{\mathrm{d} U}{\mathrm{~d} x}=-\frac{B}{4 \pi} \frac{\mathrm{d} B}{\mathrm{~d} x}
$$

which may be integrated to give

$$
F U+\frac{B^{2}}{8 \pi}=\Pi \text {. }
$$

Here the constant of integration, $\Pi$, is another of the principal integrals of the motion. It may be regarded as having the physical significance of the sum of the stagnation pressure and the magnetic pressure and as being the momentum flux density.

Next from (6) write

$$
\dot{B} \equiv U \frac{\mathrm{d} B}{\mathrm{~d} x}=4 \pi(e / c)\left(F / m_{\mathrm{t}}\right)\left(w_{\mathrm{i}}-w_{\mathrm{e}}\right) .
$$

Differentiate this with respect to $t$, using (8) to eliminate $\dot{w}_{n}$, and get

$$
\begin{aligned}
\ddot{B} & =\frac{4 \pi e^{2} F}{c^{2} m_{\mathrm{i}} m_{\mathrm{e}}}(-E c+U B) \\
& =\frac{4 \pi e^{2}}{c^{2} m_{\mathrm{i}} m_{\mathrm{e}}}\left(-F E c+\Pi B-\frac{B^{3}}{8 \pi}\right) .
\end{aligned}
$$

We now see how much the use of a dot as an abbreviation for $U \frac{\mathrm{d}}{\mathrm{d} x}$ clarifies the analysis.

If we multiply (7) by $m_{p} / q_{p}$ and sum over $p$, using (9) and (12), we get for the electric field in the $x$-direction

$$
E_{x}(x)=\frac{m_{\mathrm{i}}-m_{\mathrm{e}}}{e} \dot{U}=-\frac{m_{\mathrm{i}}-m_{\mathrm{e}}}{4 \pi e F} B \dot{B} .
$$

The electrostatic potential, $\Theta(x)$, whose negative gradient gives $E_{x}$ is

$$
\Theta(x)=-\frac{m_{\mathrm{i}}-m_{\mathrm{e}}}{2 e} U^{2}+\text { const . }
$$

(There is, of course, an additional term in the complete electrostatic potential associated with the constant field $-E e_{z}$.) Since in all real situations $m_{\mathrm{i}} \gg m_{\mathrm{e}}$, (17) states that for the ions the change in potential energy due to changes in $\Theta$ is almost precisely balanced by changes in the kinetic energy as- 
sociated with the $x$-component of the velocity. For the electrons, on the other hand, with their opposite charge and small mass, there will be no approximate balance between changes in $-e$ and $\frac{1}{2} m_{\mathrm{i}} U^{2}$. Instead conservation of energy will be maintained by the large amounts of kinetic energy associated with the high velocities in the $z$-direction required by (19) and by large potential energies associated with motion in the field $-E \boldsymbol{e}_{z}$.

If $z_{\mathrm{i}}$ and $z_{\mathrm{e}}$ are the displacements in the $z$-direction of any particular ion and electron, each measured from its own conveniently defined averaged straight trajectory, and $\dot{z}_{p}=w_{p}$, then in view of (9) it is convenient to introduce a quantity $z_{\mathrm{r}}$ (which is almost the negative of the displacement of the electron) and a reduced mass $m_{\mathrm{r}}$ defined by

$$
\begin{gathered}
m_{\mathrm{r}}{ }^{-1}=m_{\mathrm{i}}^{-1}+m_{\mathrm{e}}^{-1}, m_{\mathrm{r}} z_{\mathrm{r}}=m_{\mathrm{i}} z_{\mathrm{i}}=-m_{\mathrm{e}} z_{\mathrm{e}}, \\
z_{\mathrm{r}}=z_{\mathrm{i}}-z_{\mathrm{e}} .
\end{gathered}
$$

The corresponding velocity is

$\dot{z}_{\mathrm{r}}=w_{\mathrm{r}}=w_{\mathrm{i}}-w_{\mathrm{e}}, m_{\mathrm{r}} w_{\mathrm{r}}=m_{\mathrm{i}} w_{\mathrm{i}}=-m_{\mathrm{e}} w_{\mathrm{e}}$.

This enables us to integrate (14), getting

$$
B-B^{*}=\frac{4 \pi e F}{m_{\mathrm{t}} c} z_{\mathrm{r}}
$$

where $B^{*}$, the constant of integration, is a suitable defined average field. Thus (15) can easily be con- verted into an equation for $z_{r}$ and hence can be regarded as an equation for the particle trajectories as well as an equation for the magnetic field. And the entire subsequent analysis may be regarded as an analysis of trajectories or of the magnetic field.

Equation (15) may be regarded as the equation for a one-dimensional anharmonic oscillator, which suggests the formation of the energy integral and the determination of the solution from this integral. Thus (15) may be written

$$
\begin{gathered}
\frac{m_{\mathrm{i}} m_{\mathrm{e}} c^{2}}{16 \pi^{2} e^{2} F}=-\frac{\mathrm{d} \Phi}{\mathrm{d} B}, \\
\Phi(x)=\frac{E c B}{4 \pi}+\frac{1}{2} F U^{2}
\end{gathered}
$$

where (13) is used to express $U$ in terms of $B$. The desired integral is

$$
\frac{m_{\mathrm{i}} m_{\mathrm{e}} c^{2}}{32 \pi^{2} e^{2} F} \dot{B}^{2}+\Phi(B)=\Psi .
$$

If one wishes to express the equations of motion in terms of mean densities, one can eliminate $F, E$, and $\Pi$ by using equs. (68), (69), and (70) below. The physical significance of the constant of integration, $\Psi$, is most evident if it is expressed in terms of $z_{p}$ and $w_{p}$ or $j$ by means of $(18)-(20)$. This gives

$$
\Psi=\frac{F}{m_{\mathrm{t}}}\left[\frac{1}{2}\left(m_{\mathrm{i}}+m_{\mathrm{e}}\right) U^{2}+\frac{1}{2}\left(m_{\mathrm{i}} w_{\mathrm{i}}^{2}+m_{\mathrm{e}} w_{\mathrm{e}}^{2}\right)\right]+\frac{B E c}{4 \pi}=N U\left(\frac{1}{2} m_{\mathrm{t}} U^{2}+\frac{1}{2} m_{\mathrm{r}} w_{\mathrm{r}}^{2}\right)+\frac{B E c}{4 \pi}
$$

Now $\left(F / m_{\mathrm{t}}\right)$ is the particle flux and the quantity in square brackets is the energy per ion-electron pair. Hence $\Psi$ is just the energy flux convected by the particles plus the Poynting flux in the $x$-direction, $B E c / 4 \pi$. One must not include in the square bracket any term for the potential energy of the particles in the electric field since all electromagnetic energy is regarded as given instead by $\left(B^{2}+E^{2}\right) / 8 \pi$, and all electromagnetic energy flux by the Poynting vector. $\Psi$ is the last of the integrals of the motion necessary to specify the nature of the system and at the same time the particular oscillations it is carrying out.

\section{Reduction to dimensionless form}

The number of parameters on which the solution depends can be reduced from five to two by introducing new dimensionless variables and parameters in terms of which the previous variables and parameters are defined as follows:

$$
\begin{aligned}
B= & (8 \pi \Pi)^{1 / 2} b \\
U & =(\Pi / F) u \\
E & =(8 \pi \Pi)^{1 / 2}(\Pi / c F) \varepsilon \\
\Phi & =\left(2 \Pi \Pi^{2} / F\right) \varphi \\
\Psi \quad= & \left(2 \Pi^{2} / F\right) \psi \\
\Omega_{0}^{2}= & 4 \pi e^{2} \Pi / c^{2} m_{\mathrm{i}} m_{\mathrm{e}} \\
t & =\tau / \Omega_{0} \\
x \quad= & \left(\Pi / F \Omega_{0}\right) \xi \\
z_{\mathrm{r}}= & \left(\Pi / F \Omega_{0}\right) \zeta \\
E_{x}= & (8 \pi \Pi)^{1 / 2}(\Pi / c F) \\
& \cdot\left(m_{\mathrm{i}}-m_{\mathrm{e}}\right)\left(2 m_{\mathrm{i}} m_{\mathrm{e}}\right)^{-1 / 2} \varepsilon_{x} \\
\Theta \quad= & \left(m_{\mathrm{i}}-m_{\mathrm{e}}\right) e^{-1}(\Pi / F)^{2} \vartheta \\
w_{\mathrm{r}}= & (\Pi / F) \zeta^{\prime}
\end{aligned}
$$

Differentiation with respect to $\tau$ will be denoted by a prime and means $\mathrm{d} u / \mathrm{d} \xi$. The physical signi- 
ficance of $\Omega_{0}{ }^{2}$ is that it is the product of the gyrofrequencies of an ion and of an electron in a magnetic field whose "pressure", $B^{2} / 8 \pi$, is $\frac{1}{2} \Pi$. The most important of the new variables are $b$, which is the dimensionless magnetic field, and $u$, the dimensionless velocity in the $x$-direction.

In terms of the new variables, equs. (13), (21), (22), (23), (11), (16), and (17) become

$$
\begin{array}{ll}
u & =1-b^{2}, \\
b^{\prime \prime} & =-\varepsilon+u b=-\varepsilon+b-b^{3}=-\mathrm{d} \varphi / \mathrm{d} b, \\
\varphi(b) & =\varepsilon b+\frac{1}{4} u^{2}, \\
\frac{1}{2} b^{\prime 2}+\varphi & =\psi, \\
b^{2} & \leqq 1 \\
\xi^{\prime} & =u, \\
b^{\prime} & =\left(m_{\mathrm{r}} / 2 m_{\mathrm{t}}\right)^{1 / 2} \zeta^{\prime}, \\
\varepsilon_{x} & =-\mathrm{d} \vartheta / \mathrm{d} \xi=u^{\prime}, \\
\vartheta & =\frac{1}{2}\left(u_{\mathrm{m}}^{2}-u^{2}\right)
\end{array}
$$

where $u_{\mathrm{m}}$ is the minimum value of $u$, i. e. the value where $b$ is a maximum, which is the convenient point at which to take the zero of potential.

An important feature of the numbered reduced equations is that the parameter $m_{\mathrm{e}} / m_{\mathrm{i}}$ does not occur, and hence the solution is independent of this mass ratio provided one finds $b$ and not $\zeta$.

\section{Solution of the differential equations}

Most of the important properties of the solution of this set of equations may be understood from Fig. 1, which shows the "potential" $\varphi(\varepsilon, b)$ for $\varepsilon=0$

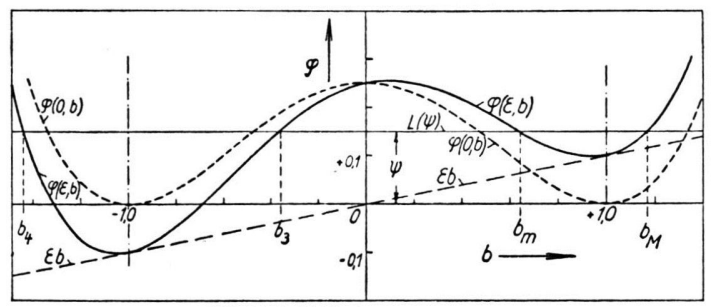

Fig. 1. $\varphi(\varepsilon, b)$ as a function of $b$ for $\varepsilon=0$ and $\varepsilon=0.1$. The intersections with the line $L(\psi)$ at $\varphi=\psi=0.15$ determine the roots $b_{\mathrm{M}}, b_{\mathrm{m}}, b_{3}$, and $b_{4}$. Condition (40) is not satisfied since $b_{M}>1$.

and for some convenient value of $\varepsilon$ between 0 and $2 \sqrt{3} / 3$. Note that $\varphi(0, b)$ has a maximum at $b=0$ and minimum at $b= \pm 1$. The positive directions of the coordinate axes were so defined that $\varepsilon>0$ and $u>0$. Thus $\varphi(\varepsilon, b)$, which is obtained from $\varphi(0, b)$ by adding the linear term $\varepsilon b$, has one minimum for $b<-1$ and, if $0<\varepsilon<2 \sqrt{3} / 3$, first a maximum and then a minimum as one goes from $b=0$ to $b=1$. If we draw a horizontal line, $L(\psi)$, at $\varepsilon=\psi$, we see from (37) and (39) that the variation of $b$ with $\tau$ is just that of a unit mass oscillating between the points at which $L(\psi)$ intersects $\varphi(\varepsilon, b)$ in the potential trough $\varphi(\varepsilon, b)$. It is at once clear that the only solutions satisfying (40) are those for which: first, $\varepsilon$ is small enough so that there are two minima, the oscillation taking place in the one for which $b>0$; (since if one starts to the left one must go at least to the minimum, where $\left.b^{2}>1\right)$; and second, $\psi$ is in the range for which $L(\psi)$ intersects $\varphi(b)$ four times, the maximum value of $b$ at an intersection being $\leqq 1$. The variation with time of $b$ and $u$ is the variation experienced by any particular particle as it travels through the wave pattern. The wave pattern in space is relatively easily found from the time variations.

Except for a trivial phase constant, each possible solution is characterized by the values of $\varepsilon$ and $\psi$, which determine the roots of $\psi-\varphi=0$. However, it is more convenient to characterize the solutions by parameters that give the roots, and $\varepsilon$ and $\psi$, without requiring the solution of a fourth order equation. If the roots are

$$
b_{4}<b_{3} \leqq b_{\mathrm{m}} \leqq b_{\mathrm{M}}
$$

as shown in Fig. 1, convenient parameters are $c$ and $\mathfrak{b}$ defined by

$$
b_{\mathrm{M}}=\mathfrak{b}+a, \quad b_{\mathrm{m}}=\mathfrak{b}-a .
$$

Thus $a$ is the amplitude of the oscillation and $\mathfrak{b}$ its medium value. Next define the polynomial

$$
\begin{aligned}
P(b) & =4[\psi-\varphi(\varepsilon, b)]=-b^{4}+2 b^{2}-4 \varepsilon b+4 \psi-1 \\
& =(-b+\mathfrak{b}+a)(b-\mathfrak{b}+a)\left(b-b_{3}\right)\left(b-b_{4}\right) .
\end{aligned}
$$

If we expand and equate coefficients of powers of $b$, any two of $\varepsilon, \psi, \mathfrak{b}, a, b_{3}, b_{4}$ determine the other four. Since we take $a$ and $\mathfrak{b}$ as basic, we get

$$
\begin{aligned}
\varepsilon & =\mathfrak{b}\left(1-\mathfrak{b}^{2}-a^{2}\right), \\
\psi & =\frac{1}{4}\left[1+\left(\mathfrak{b}^{2}-a^{2}\right)\left(2-3 \mathfrak{b}^{2}-a^{2}\right)\right], \\
b_{3} & =-\mathfrak{b}+R, \quad b_{4}=-\mathfrak{b}-R
\end{aligned}
$$$$
\text { with } \quad R=\left(2-2 \mathfrak{b}^{2}-a^{2}\right)^{1 / 2} \text {. }
$$

Equations (46) and (47) have been used to construct Fig. 3, from which $(\varepsilon, \psi)$ may be found if $(\mathfrak{b}, a)$ are given. 
Equation (39) can now be solved to give

$$
\tau(b)=\sqrt{2} \int_{b}^{\mathfrak{b}+a} \frac{\mathrm{d} b}{[P(b)]^{1 / 2}}
$$

where we have fixed the phase by taking $\tau=0$ at a maximum of $b$. This equation is in the standard form for solution in term of elliptic integrals by formula BF 257.00 of Byrd and Friedman ${ }^{2}$. The result is expressed in terms of

$$
\begin{aligned}
g & =\left[2 /\left(3 \mathfrak{b}^{2}+R a-1\right)\right]^{1 / 2}, \\
k^{2} & =a R g^{2}, \\
-a^{2} & =2 a /(2 \mathfrak{b}-a+R) .
\end{aligned}
$$

The period, $\tau_{\mathrm{P}}$, and angular frequency, $\omega$, are given by

$$
\omega=2 \pi / \tau_{\mathrm{P}}=g K(k) \pi / \sqrt{2}
$$

where $K(k)$ is the usual complete elliptic integral of the first kind. With the aid of Fig. 4 and (79) below, it is easy to determine the approximate value of $w$ as a function of $(\mathfrak{b}, a)$. The variation of $b$ with $\tau$ is given by

$$
b=\frac{\mathfrak{b}+a-b_{4} a^{2} \operatorname{sn}^{2}(\sqrt{2} \tau / g)}{1-a^{2} \operatorname{sn}^{2}(\sqrt{2} \tau / g)}
$$

where $\operatorname{sn}(u, k)$ is the usual JAcobian elliptic function. Equation (36) now gives $u$. With the aid of $\mathrm{BF} 336$ and BF 337 it is then possible to express $\xi(\tau), b(\xi)$, and any other quantities of interest in terms of complicated formulas involving elliptic functions and integrals of the first, second, and third kinds.

These methods become very laborious if one wants more than a few isolated results. Accordingly we have solved the differential equations (36) to (43) numerically in 32 cases, using the G 2 digital computer of the Max-Planck-Institut für Physik. The general procedure was to evaluate $\tau_{\mathrm{P}}$ from (54), to integrate (37) by the STöRmer procedure over half a period starting at a maximum in $b$, and to evaluate a number of subsidiary quantities and integrals. The accuracy of the calculation was checked by requiring that the final value of $b$ had to differ from $\mathfrak{b}-a$ by less than $0.01 \%$ (except that in one case it was $0.05 \%$ ) and that the period had to be right. These results, supplemented by hand computation of some limiting cases, were used to con-

2 Byrd and Friedman, Handbook of Elliptic Integrals for Engineers and Physicists, Springer, Berlin 1954. Their formula No. 257.00 will be referred to as BF 257.00 , etc.

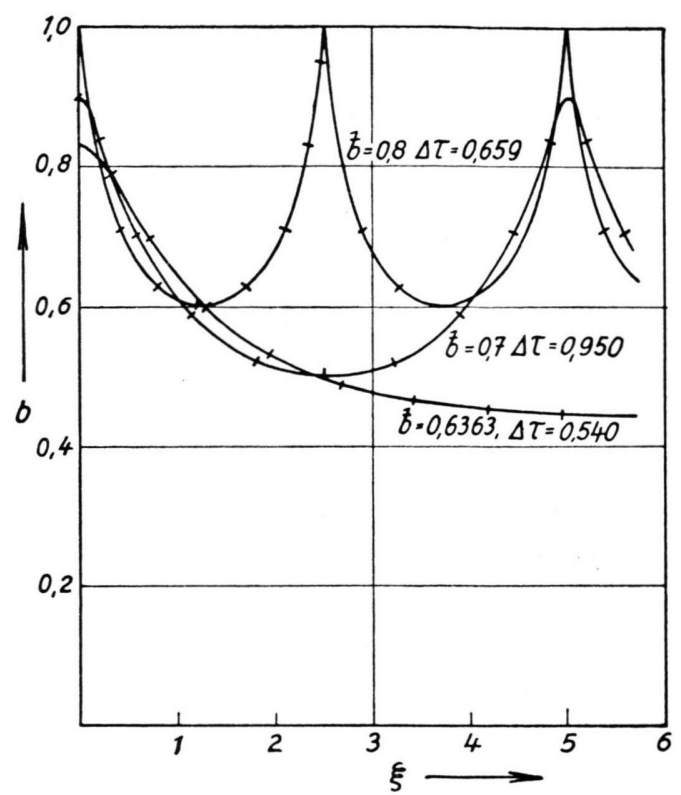

Fig. 2 a.

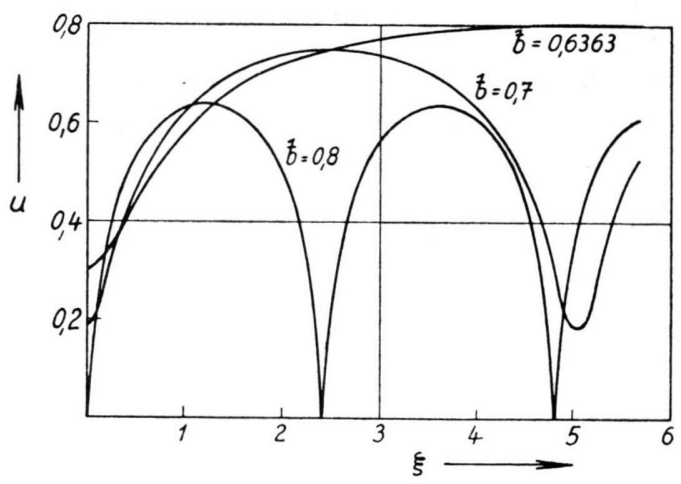

Fig. 2 b.

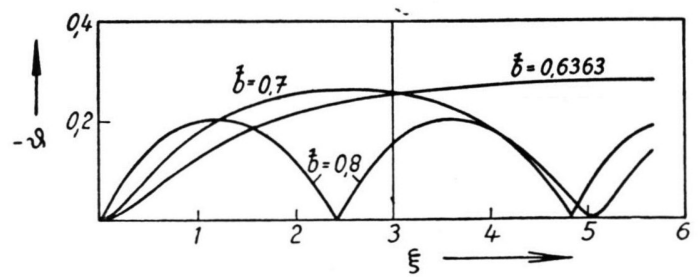

Fig. 2 c.

Fig. 2. The waves in space for $a=0.2, \mathfrak{b}=0.8,0.7$, and 0.6363 . The solitary wave, $\mathfrak{b}=0.6363$, has infinite wavelength. Fig. $2 \mathrm{a}$ shows $b$ as a function of $\xi$ and hence, by (20), the shape of the particle trajectories. The marks on each curve show the points reached at equal intervals of dimensionless time, $\Delta \tau$, the value of $\Delta \tau$ being given for each curve. Figs. $2 \mathrm{~b}$ and $2 \mathrm{c}$ show $u$ and $-\vartheta$, respectively as functions of $\xi$. 


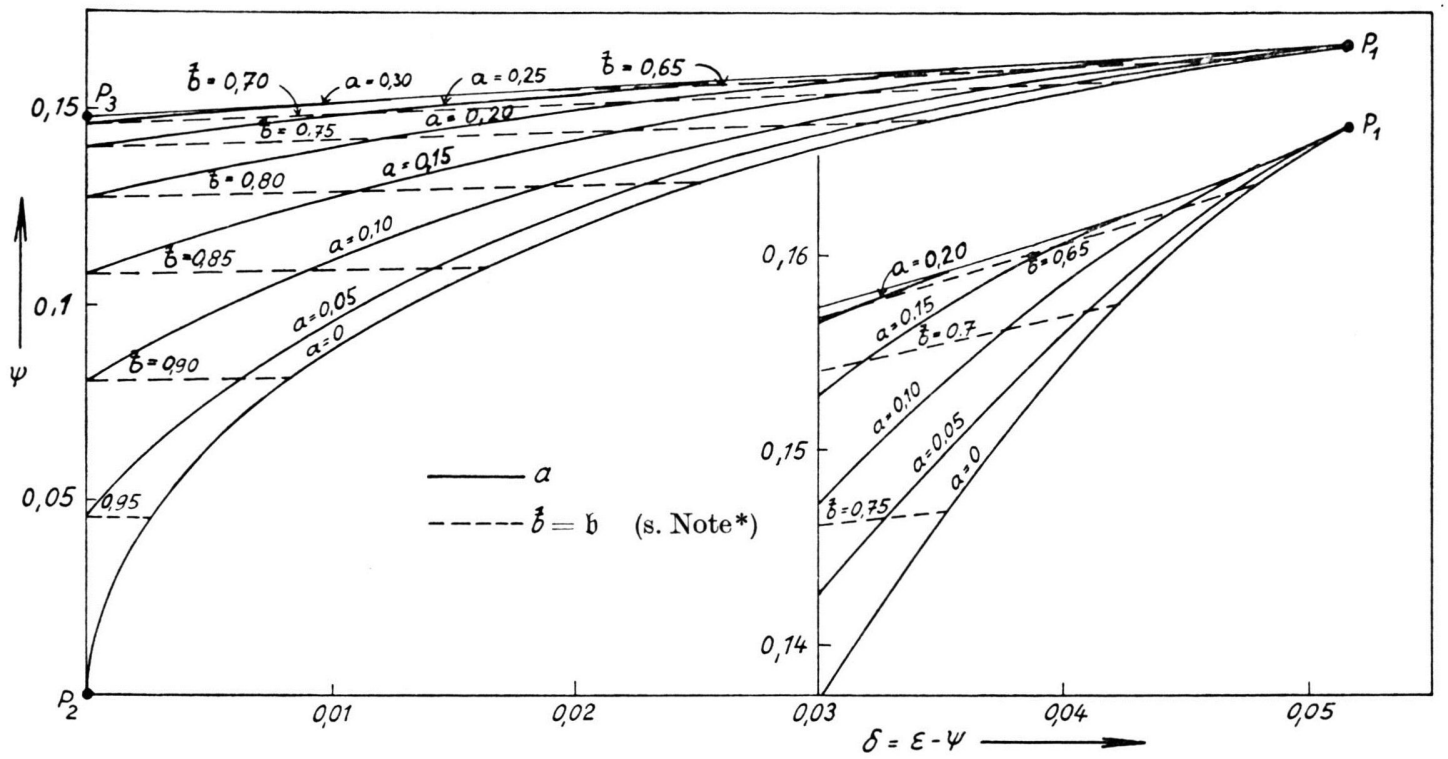

Fig. 3. The dependence of $(\varepsilon, \psi)$ on $(\mathfrak{b}, a)$. The points $\mathrm{P}_{1}, \mathrm{P}_{2}, \mathrm{P}_{3}$ come at values of $(\mathfrak{b} a)$ of $(1 / \sqrt{3}, 0),(1,0),\left(\frac{2}{3}, \frac{1}{3}\right)$, respectively, and at values of $(\varepsilon, \psi)$ of $\left(\frac{2}{3} \sqrt{3}, \frac{1}{3}\right),(0,0)$, and $(8 / 27,8 / 27)$, respectively. The detail near $\mathrm{P}$, is shown at the right by using a different scale for $\psi$. Curves on which $a$ is constant are shown by solid lines, curves for constant $b$ by dashed lines.

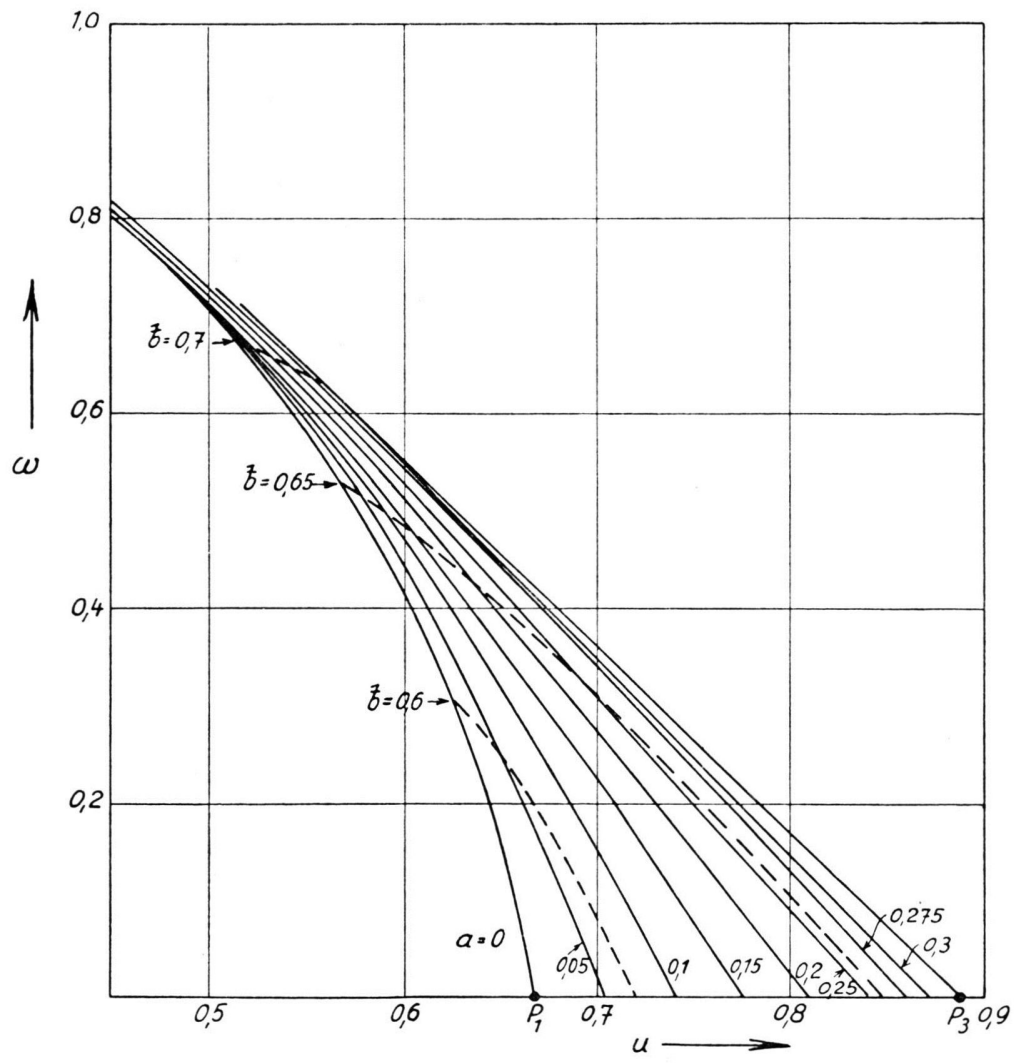

* Note added in press: The letter $\bar{b}$ in the Figs. $2-4$ stands for the letter $\mathfrak{b}$ in the text.

Fig. 4. The dependence of frequency, $\omega$, and the average velocity of the wave, $\bar{u}$, on $(\mathrm{b}, a)$. Only part of the range is shown because elsewhere the curves crowd together, and (81) and (82) are quite accurate. The points $\mathrm{P}_{1}, \mathrm{P}_{2}, \mathrm{P}_{3}$ come at values of $(\bar{u}, \omega)$ of $\left(\frac{2}{3}, 0\right),(0, \sqrt{2}),(8 / 9,0)$ respectively.

struct Figs. 3-6 which summarize the properties of these waves. Some wave forms for high amplitude waves are shown in Fig. 2.
Fig. 3 shows $\psi$ plotted as a function of $\delta=\varepsilon-\psi$ for various values of $\mathfrak{b}$ and of $a, \delta$ being used as an ordinate in order to spread the curves out more. 
Each point determines the value of the pair $(\mathfrak{b}, a)$ and of the pair $(\varepsilon, \psi)$ and hence enables one to find the value of either pair when the other is known. In the same way Fig. 4 gives $(\bar{u}, \omega)$ as functions of $(\mathfrak{b}, a)$. Here $\omega$ is the frequency of the wave and $\bar{u}$, defined precisely by (57) below, is the average value of $u$ and hence the velocity of the wave through the material in the dimensionless units defined by (26). Only part of the range of possible values is shown because over the rest of the range all the curves crowd together and (79) below is a very good approximate formula. Fig. 5 gives $\left(\bar{b}, \vartheta_{\mathrm{T}}\right)$, where $\vartheta_{\mathrm{T}}$ is the dimensionless potential difference between the trough and the crest of the waves and $\bar{b}$ is the time average of the dimensionless magnetic field, defined precisely by (57) below. Although $\bar{b}$ is in itself of not too much interest, it is used in the formulas to be developed for more important averages. Fig. 6 shows the relative amplitude of the wave and $\mathrm{M}_{\mathrm{ACH}}$ number [defined by (76)] as functions of $(\mathfrak{b}, a)$. Its main use is in section 7 for the conversion from the "flux" characterization of the motion to the "mean density" characterization.

It will be noted that in these figures only a limited range of the variables is allowed, and it is of interest to consider the various boundaries. Along the line $\mathrm{P}_{1} \mathrm{P}_{2}, a=0$ and we have waves of zero amplitude. In the neighborhood of this line the linear approximation is satisfactory; and, by including the low order non-linear terms as in section 8, the range of usefulness of the approximate formulas can be considerably extended. The boundary line $\mathrm{P}_{2} \mathrm{P}_{3}$ results from (40). Along this line $u=0$ at the maximum value of $b$ and the waves have cusps at their crests. However, the equations to be solved have no particular singularities as long as $\tau$ is used as the independent variable. The line is defined by $\mathfrak{b}+a=1$ or by $\psi=\varepsilon$ as one sees from (36), (38), and (39). Along the curve $\mathrm{P}_{1} \mathrm{P}_{3}$ the two middle roots of $P(b)$ coincide, $L(\psi)$ is tangent to the top of the maximum in Fig. 1, and larger values of $\psi$ for this $\varepsilon$ are not allowed. Thus the oscillation goes just to the top of the maximum in Fig. 1 and must have an infinite period. This is a solitary wave. There is just one crest in the entire system and each particle remains balanced at $b=b_{\mathrm{m}}$ at the top of the potential maximum except when near this crest. The condition to be fulfilled along $\mathrm{P}_{1} \mathrm{P}_{3}$ is that $b_{3}=b_{\mathrm{m}}$, or by (44) and (48)

$$
3 \mathfrak{b}^{2}-2 a \mathfrak{h}+a^{2} \geqq 1,
$$

equality defining the line. Solitary waves are treated further in section 9 .

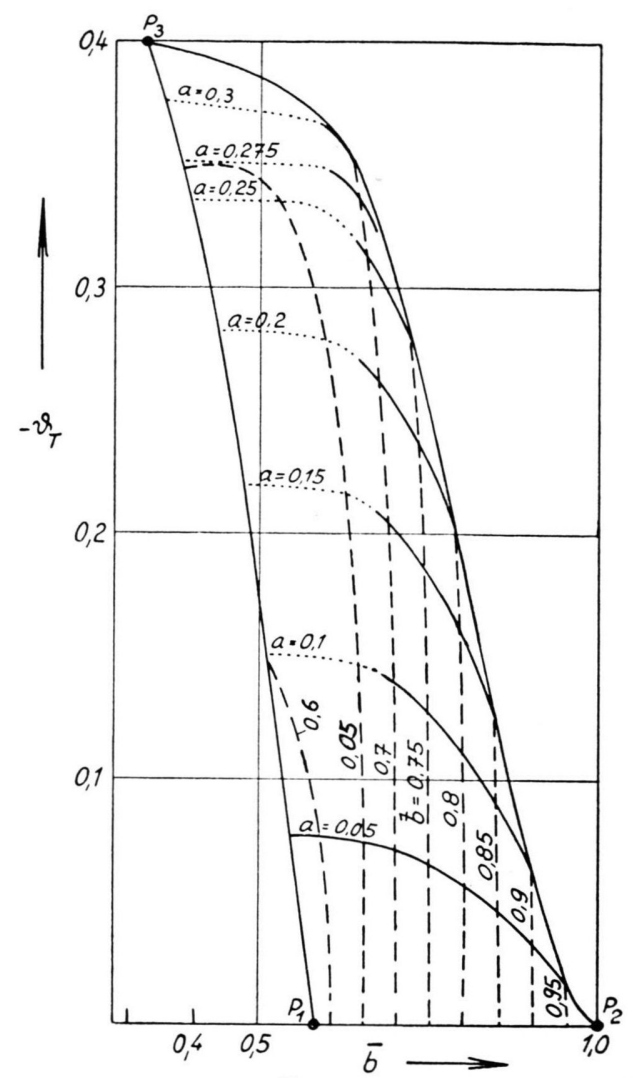

Fig. 5. The dependece of $\bar{b}$, the time average of $b$, and $-\vartheta_{\mathrm{T}}$, the total potential difference between the crest and trough of the wave, on $(\mathfrak{b}, a)$. The points $\mathrm{P}_{1}, \mathrm{P}_{2}, \mathrm{P}_{3}$ come at values of $(\bar{b}, \omega)$ of $(1 / \sqrt{3}, 0),(1,0),\left(\frac{1}{3}, \frac{3}{8} \frac{2}{1}\right)$, respectively.

For the limiting case in which the amplitude of the waves is zero there is no prefered reference system and any inertial system may be used. No problems arise in the density characterization, but $\Pi$ and $\Psi$ of the flux characterization do not vary linearly with the velocity of the reference system and this complicates the connections between the dimensionless parameters and the properties of the waveless flow. This problem is treated in section 10 . However, most quantities of interest are given by the curves. When the $\mathrm{MACH}_{\mathrm{ACH}}$ number, $M$, defined by (76) below, is in the range $M \leqq 1$, the curve $\mathrm{P}_{1} \mathrm{P}_{2}$ for $a=0$ applies and $\mathfrak{b}$ is just the dimensionless magnetic field. If one wishes to use a frame of reference with respect to which the velocity is so high that $M \geqq 1$, one uses the bounding curve $\mathrm{P}_{1} \mathrm{P}_{3}$ for the solitary wave case. For such a perfectly waveless flow may be regarded as part of an infinitely distant 
solitary wave. However the values of $a$ and $\mathfrak{b}$ shown along $\mathrm{P}_{1} \mathrm{P}_{3}$ apply to the crest of the solitary wave and for the smooth part the field is $\mathfrak{b}-a$.

Since $k$, the modulus of the elliptic functions and integrals defined by (52), approaches 0 as one approaches $\mathrm{P}_{1} \mathrm{P}_{2}$ and approaches 1 as one approaches $P_{1} P_{3}$, the point $P_{1}$ is in many ways a singular point. It corresponds to the situation in which $b_{n}$, $b_{m}$, and $b_{3}$ coincide in Fig. 1 , the minimum and maximum coalescing in a horizontal inflection point. Thus the motion may depend in a sensitive way on the parameters and in some of the figures the curves either all come together at $\mathrm{P}_{1}$ or the curves for $a$ and $\mathfrak{b}$ become parallel there. As we shall see below, if $a$ approaches zero fast enough as one approaches $\mathrm{P}_{1}$, the waves approach the usual long compressional magneto-acoustic waves which move with the Alfvén velocity and can have arbitrary shape.

\section{Formulas for average values}

It is useful to consider two kinds of averages, time averages and space averages, defined, respectively, by

$$
\begin{gathered}
\overline{f(b, u)}=\frac{1}{\tau_{\mathrm{P}}} \int_{0}^{\tau_{\mathrm{P}}} f(b, u) \mathrm{d} \tau, \\
\langle f(b, u)\rangle=\frac{1}{\xi_{\mathrm{P}}} \int_{0}^{\xi_{\mathrm{P}}} f(b, u) \mathrm{d} \xi \\
=\frac{1}{\bar{u} \tau_{\mathrm{P}}} \int_{0}^{\tau \mathrm{P}} f(b, u) u \mathrm{~d} \tau=\frac{[u f(b, u)]}{\bar{u}}
\end{gathered}
$$

where $\tau_{\mathrm{P}}$ is the period, $\xi_{\mathrm{P}}=\tau_{\mathrm{P}} \bar{u}$ is the distance traveled in one period, and (41) is used to convert an integral over $\xi$ to one over $\tau$. The time averages defined by (57) can also be regarded as equal to space averages in which one uses a weighting factor proportional to the density, $1 / u$, and hence as equal to the average over all the particles. Since equation (36) enables us to express all functions of $u$ as functions of $b$, and (58) reduces space averages to time averages, the average of any polynomial function of $u$ and $b$ can be expressed in terms of $\overline{b^{n}}$. Hence we next establish the recursion formula

$$
\begin{aligned}
\overline{b^{n+2}}= & \frac{2 n}{(n+1)} \overline{b^{n}}-\frac{2(2 n-1)}{(n+1)} \varepsilon \overline{b^{n-1}} \\
& \quad+\frac{(n-1)}{(n+1)}(4 \psi-1) \overline{b^{n-2}}, \quad n>0
\end{aligned}
$$

which enables us to calculate all $\overline{b^{n}}$ in terms of $\overline{b^{0}}=1, \bar{b}$, and $\overline{b^{2}}=1-\bar{u}$. These are given by Figs. 4 and 5 or may, if necessary, be computed from elliptic integrals.

To establish (59), one starts from the identity

$$
\frac{\mathrm{d}^{2} b^{n}}{\mathrm{~d} \tau^{2}}=b^{n-1} b^{\prime \prime}+2(n-1) b^{n-2} b^{\prime 2}+b \frac{\mathrm{d}^{2} b^{n-1}}{\mathrm{~d} \tau^{2}}
$$

and expresses $b^{\prime \prime}$ and $b^{\prime}$ by means of (37) and (39). It then follows by induction that

$$
\begin{aligned}
\frac{\mathrm{d}^{2} b^{n}}{\mathrm{~d} \tau^{2}}= & -\frac{1}{2} n(n+1) b^{n+2}+n^{2} b^{n}-n(2 n-1) \varepsilon b^{n-1} \\
& +\frac{1}{2}(n-1) n(4 \psi-1) b^{n-2}, \quad n>0 . \quad(60)
\end{aligned}
$$

If this is integrated with respect to $\tau$ from 0 to $\tau_{\mathrm{P}}$, one gets (59).

The averages of greatest interest are perhaps $\bar{u}$, $\langle b\rangle$, and the averages that give the kinetic energy. Since $\bar{u}$ is the average dimensionless velocity of the particles in the frame of reference in which the wave stands still, it is also the average velocity of the wave with respect to the frame of reference in which the particle have zero average velocity. If one wished to know the dimensionless uniform magnetic field whose flux through any large region is the same as that in the wave, the appropriate average field is

$$
\langle b\rangle=\frac{\overline{b u}}{\bar{u}}=\frac{\varepsilon}{u},
$$

as one sees from (59) or by averaging (37) over $\tau$. Thus $\varepsilon=\bar{u}\langle b\rangle^{-}=(\overline{b u})$. The space average of the kinetic energy density associated with motion in the $x$-direction is

$$
\begin{gathered}
\frac{1}{2}\left\langle m_{\mathrm{t}} N U^{2}\right\rangle=\frac{1}{2}\langle F U\rangle=\frac{1}{2} \Pi\langle u\rangle=\frac{1}{2} \Pi \bar{u}^{2} / \bar{u} \\
=\Pi(2 \psi-3 \varepsilon \bar{b}-\bar{u}) / 3 \bar{u} .
\end{gathered}
$$

That associated with motion in the $z$-direction is

$$
\begin{aligned}
& \frac{1}{2}\left\langle m_{\mathrm{i}} N w_{\mathrm{i}}{ }^{2}+m_{\mathrm{e}} N w_{\mathrm{e}}{ }^{2}\right\rangle=\frac{F m_{\mathrm{r}}}{2 m_{\mathrm{t}}}\left\langle\frac{w_{\mathrm{r}}{ }^{2}}{U}\right\rangle \\
& =\Pi\left\langle b^{\prime 2} / u\right\rangle=2 \Pi(\psi-\bar{\varphi}) / \bar{u}=\Pi(2 \psi-3 \varepsilon \bar{b}-\bar{u}) / 3 \bar{u}
\end{aligned}
$$

where we have used (19) to introduce $w_{\mathrm{r}},(20)$ to express it in terms of $B,(25)-(31)$ to put the result in dimensionless form, and (39), (38), and (59) to get the final form.

\section{The connections between mean densities and the fluxes}

The system and its state of motion are now completely specified, except for a trivial phase constant, in terms of $E, \Psi, \Pi$, and $F$, (and of course $\Omega_{0}$, which depends on the atomic constants and $F$ ) or in terms of $\varepsilon, \psi, \Pi, F$, and $\Omega_{0}$. As discussed in sec- 
tion 1, this "flux characterization" has many advantages. On the other hand there are cases where a more direct approach is preferable and one wishes to describe the system in terms of the mean particle density $N_{0}$, and the mean density of magnetic flux, $B_{0}$, and to describe the waves in terms of their amplitude, $A$, and velocity, $U_{0}$. Of course it does not really matter which method of specification is used. In either case one can write the equations of motion, can solve them (numerically at least) for the particular solution having the desired average densities or fluxes, and from this solution compute all the other properties that could be used for the alternative specification of the system and its motion. What are now needed are formulas that enable one to convert from either mode of specification to the other so that the more convenient can always be used.

It seems natural to define some of the average densities in terms of space and some in terms of time averages. This suggests the use of the notation of the last section except that $\tau_{\mathrm{P}}$ and $\xi_{\mathrm{P}}$ are replaced by $t_{\mathrm{P}}$ and $x_{\mathrm{P}}$, respectively; i.e. the period and displacement in a period expressed in dimensional units. The two kinds of averages are still easily connected by a formula similar to (58). Define the average magnetic field as

$$
B_{0}=\frac{1}{x_{\mathrm{P}}} \int_{0}^{x_{\mathrm{P}}} B(x) \mathrm{d} x=\langle B\rangle .
$$

The average velocity of a particle through the stationary system of waves, and hence the equal but opposite velocity of the waves through a medium that is, on the average, stationary is given by

$$
U_{0}=\frac{1}{t_{\mathrm{P}}} \int_{0}^{t_{\mathrm{p}}} U(t) \mathrm{d} t=\bar{U}
$$

where of course the average over any very long interval is just the average over one period. The average particle density; i.e. density of ion-electron pairs, is

$$
N_{0}=\frac{1}{x_{\mathrm{P}}} \int_{0}^{x_{\mathrm{P}}} N(x) \mathrm{d} x=\langle N\rangle .
$$

The average mass density is $\varrho_{0}=m_{\mathrm{t}} N_{0}$.

Given $N_{0}, U_{0}, B_{0}$, and $A$, the amplitude, the motion is determined and one can, at least in principle, obtain the solution and from it such quantities as $B_{\mathrm{MI}}$, the maximum value of $B$, and $B^{2}$. If we convert (65) from an integral over $t$ to an integral over $x$, we get, as expected,

$$
U_{0}=\frac{1}{t_{\mathrm{P}}} \int_{0}^{x_{\mathrm{P}}} \mathrm{d} x=\frac{x_{\mathrm{P}}}{t_{\mathrm{P}}} .
$$

If we convert (66) to an integral over $t$ and use (10) we see that

$$
m_{\mathrm{t}} U_{0} N_{0}=m_{\mathrm{t}} \frac{x_{\mathrm{P}}}{t_{\mathrm{P}}} \frac{1}{x_{\mathrm{P}}} \int_{0}^{t_{\mathrm{P}}} N(t) U(t) \mathrm{d} t=F .
$$

In a similar way, if we convert (64) to an integral over $t$ and use (8), we see that

$$
\begin{aligned}
& \frac{B_{0} U_{0}}{c}=\frac{U_{0}}{x_{\mathrm{P}}} \int_{0}^{t_{\mathrm{p}}} \frac{B(t) U(t) \mathrm{d} t}{c} \\
&=\frac{1}{t_{\mathrm{P}}} \int_{0}^{t_{\mathrm{p}}}\left[\frac{m_{\mathrm{p}} \dot{w}_{\mathrm{p}}}{q_{\mathrm{p}}}+E \mathrm{~d} t\right]=E
\end{aligned}
$$

since $w_{\mathrm{p}}$ is periodic. Thus from the average density description we readily get the constants $F$ and $E$ of the flux description. If we average (13) over time we get

$$
\Pi=F U_{0}+\left(\overline{B^{2}} / 8 \pi\right) .
$$

If we evaluate (13) and (21) at the instant when $B=B_{\mathrm{M}}$ and $\dot{B}=0$, we get

$$
\Psi=\frac{c E B_{\mathrm{M}}}{4 \pi}+\frac{1}{2 F}\left(\Pi-\frac{B_{\mathrm{M}^{2}}}{8 \pi}\right)^{2} .
$$

Thus if we have a solution based on the mean density specification we can compute the fluxes and convert to the flux specification.

To go in the reverse direction, from the solution in sections 5 or 8 based on the flux specification to the mean density specification, one determines $\bar{u}, \varepsilon$, and $\psi$ from $a$ and $\mathfrak{b}$. Then, supposing one starts from $\Pi, F, a, \mathfrak{b}$, one has by $(26)$

$$
U_{0}=(\Pi / F) \bar{u}
$$

$N_{0}$ and $B_{0}$ are given by (68) and (69), and the amplitude by $\sqrt{8 \pi \Pi} a$. The connection between dimensionless frequencies, $\omega$, and actual frequencies in $\sec ^{-1}, \Omega$, and the corresponding transitions between $\tau$ and $t$ are given by

$$
\Omega=\Omega_{0} \omega .
$$

and (31). It is convenient now to transform the definition of $\Omega_{0}$ given in (30) to

$$
\Omega_{0}^{2}=\left[\frac{e^{2} B_{0}{ }^{2}}{c^{2} m_{\mathrm{i}} m_{\mathrm{e}}}\right] \frac{4 \pi \Pi}{B_{0}{ }^{2}}=\frac{\Omega_{\mathrm{G}}^{2} \bar{u}}{2 \varepsilon^{2}}
$$


where $\Omega_{\mathrm{G}}{ }^{2}$, the term in square brackets, is the product of the ion and electron gyro-frequencies in the magnetic field $B_{0}$, and $B_{0}$ is eliminated by (69).

However, one can not directly use the previously obtained solutions in terms of $b(\tau), a, \mathfrak{b}$, and the flux characterization to obtain the solution of a problem specified by given values of $B_{0}, N_{0}, U_{0}$, and $A$ in the average density characterization because it is difficult to determine $a, \mathfrak{b}$, and $\Pi$. To overcome this difficulty, one can use Fig. 6, which shows, for various values of the parameters $a=A /(8 \pi \Pi)^{1 / 2}$ and $\mathfrak{b}$,

$$
a \bar{u} / \varepsilon=A / B_{0}
$$

as a function of

$$
\frac{\bar{u}^{3}}{2 \varepsilon^{2}}=\frac{4 \pi m_{\mathrm{t}} N_{0} U_{0}^{2}}{B_{0}^{2}} \frac{U_{0}^{2}}{V_{\mathrm{A}}^{2}}=M^{2}
$$

where $V_{\mathrm{A}}$ is the velocity of the infinitely long magneto-acoustic compression waves and of ALFvÉn waves and $M$ is the corresponding $\mathrm{M}_{\mathrm{ACH}}$ number. From the values of $A, B_{0}, N_{0}$ and $U_{0}$ one locates a point in the figure and this determines $a$ and $\mathfrak{b}$. One can then use the other figures and, since

$$
\Pi=A^{2} / 8 \pi a^{2},
$$

can convert the dimensionless solution to physical variables by $(25)-(35)$. A useful check is to determine $\varepsilon$ from Fig. 3 and from (27), using $c E=U_{0} B_{0}$. In the range where it is a good approximation, the approximate solution for small amplitudes is easily expressed directly in terms of the mean density characterization.

\section{Solution in powers of the amplitude}

It is possible to solve (37) in powers of the amplitude, a power series for the frequency being obtained from the requirement that each term in the expansion be periodic. For our present needs this procedure may be simplified to the statement that it is easily verified by direct substitution that

$$
\begin{gathered}
b=\mathfrak{b}+a \cos \omega \tau-\frac{\mathfrak{b} a^{2}}{2 \omega_{0}^{2}}(1-\cos 2 \omega \tau) \\
-\frac{\left(9 \mathfrak{b}^{2}-1\right)}{32 \omega_{0}^{4}}(\cos \omega \tau-\cos 3 \omega \tau)+O\left(a^{4}\right), \\
\omega=\omega_{0}\left[1-\frac{2-\omega_{0}^{2}}{8 \omega_{0}^{4}} a^{2}+O\left(a^{4}\right)\right], \\
\omega_{0}^{2}=3 b^{2}-1
\end{gathered}
$$

is a solution that is accurate to the indicated order in $\tilde{a}$. From this all quantities of interest may be computed.

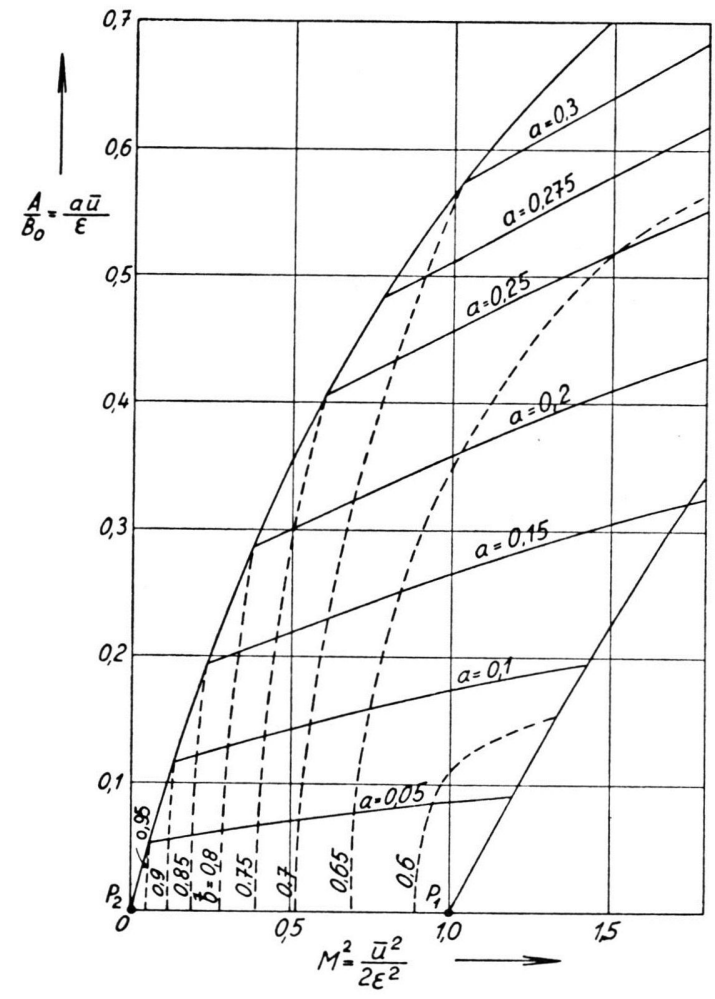

Fig. 6. The dependence of $A / B_{0}=a \bar{u} / \varepsilon$, the relative amplitude, and $M^{2}=\bar{u}^{3} / 2 \varepsilon^{2}$, the square of the $\mathrm{M}_{\mathrm{ACH}}$ number, on $(\mathfrak{b}, a)$. The points $\mathrm{P}_{1}, \mathrm{P}_{2}, \mathrm{P}_{3}$ come at values of $\left(A / B_{0}, M^{2}\right)$ of $(0,1),(0,0),(1,4)$, respectively.

In particular, the quantities plotted in Figs. $3-6$ are

$$
\begin{aligned}
& \bar{u}=\left(1-b^{2}\right)\left[1+\left(a^{2} / 2 \omega_{0}^{2}\right)+O\left(a^{4}\right)\right], \\
& \bar{b}=b\left[1-\left(a^{2} / 2 \omega_{0}^{2}\right)+O\left(a^{4}\right)\right],
\end{aligned}
$$

$M^{2}=\frac{\bar{u}^{3}}{2 \varepsilon^{2}} \frac{\left(1-b^{2}\right)}{2 b^{2}}\left[1+\frac{3\left(2+3 \omega_{0}^{2}\right)}{2 \omega_{0}^{2}\left(2-\omega_{0}^{2}\right)} a^{2}+O\left(a^{4}\right)\right]$,

$\frac{A}{B_{0}}=\frac{a \bar{u}}{\varepsilon}=\frac{a}{b}\left[1-\frac{5 \omega_{0}^{2}+2}{2 \omega_{0}^{2}\left(2-\omega_{0}^{2}\right)} a^{2}+O\left(a^{4}\right)\right]$.

One can compute $\varepsilon, \psi$, and $\vartheta_{\mathrm{T}}$ exactly by formulas which have already been given. The wave number, from which the dispersion may be found in the linear case, is

$$
\begin{aligned}
\varkappa=\frac{\omega}{\bar{u}} & =\frac{3 \omega_{0}}{2-\omega_{0}^{2}}\left[1-\frac{3 \omega_{0}^{2}+2}{8 \omega_{0}^{2}} a^{2}+O\left(a^{4}\right)\right] \\
& =\frac{3 \omega}{2-\omega^{2}}\left[1-\frac{a^{2}}{4 \omega^{2}}+O\left(a^{4}\right)\right] .
\end{aligned}
$$

Except near the solitary wave, and in particular near $P_{1}$, where $\omega_{0}^{2}=0$ and all series diverge, these approximate formulas may often be more useful than the curves for a specific calculation. The formulas should be reasonably accurate near $\mathrm{P}_{2}$, where $\tilde{a}=0, b=1$, and $\omega_{0}{ }^{2}=2$, in spite of the factor $\left(2-\omega_{0}{ }^{2}\right)$ in the denominators, but they may not be accurate to order $\tilde{a}^{4}$ in a strict sense. The accuracy of the formulas may be judg. 
ed by comparison with the results of the computations from which the curves were constructed. For $\mathfrak{b}=0.7$, $\tilde{a}=0.2$, the error in $\omega$ is only $0.1 \%$. For $b=0.7$ and $\tilde{a}=0.3$ the error in $\omega$ is only $0.6 \%$, that in $\bar{u}$ only $0.3 \%$, and that in $b$ at $\omega \tau=\pi / 2$ only $0.2 \%$. Even at $b=0.6$ and $\tilde{a}=0.05$, the error in $\bar{u}$ is only $0.2 \%$ while that in $\omega$ is $2.7 \%$. The error decreases rapidly as $b$ becomes larger or as $a$ becomes smaller.

If one is given $N_{0}, B_{0}, U_{0}$, and $A$ in the mean density characterization, one evaluates $M^{2}$ from (76) and $A / B_{0}$. Then one sets the square brackets in (83) and (84) equal to 1 and gets approximate values for $\mathfrak{b}, \tilde{a}$, and $\omega_{0}$. These approximate values are used to get better numerical values for the square brackets and hence values of $\mathfrak{b}, \tilde{a}$, and $\omega_{0}$ in which the error is $O\left(a^{4}\right)$. Finally one gets $F$ from (68) and $\Pi$ from (72) or (77). If one is given $N_{0}, B_{0}, \Omega$, and $A$, where $\Omega$ is the frequency in $\sec ^{-1}$, one gets $\omega_{0}, \mathfrak{b}$, and $\tilde{a}$ in the same way from (84) and

$$
\Omega=\frac{\Omega_{\mathrm{G}} \omega_{0}}{\left[2\left(\omega_{0}^{2}+1\right) / 3\right]^{1 / 2}}\left[1-\frac{21 \omega_{0}^{4}+4 \omega_{0}^{2}+4}{8 \omega_{0}^{4}\left(2-\omega_{0}^{2}\right)} a^{2}+O\left(a^{4}\right)\right]
$$

which is derived from (73) by the use of (74), (79), and (84). After evaluating $\bar{u}$ from (81) and $M^{2}$ from (83), one can use (77) to get $\Pi,(76)$ to get $U_{0}$, and either $(72)$ or $(68)$ to get $F$. Since there are so many equations involving these quantities, it may be possible to devise other, more efficient procedures.

If one wishes a solution in powers of $A$ in which the parameters are those of the mean density characterization and is not interested in the connections with the flux characterization, it is probably easiest to develope the series solution afresh starting from (21), which may be written as

$$
\ddot{B}=\Omega_{\mathrm{G}}{ }^{2} M^{2} U_{0}^{-1}\left[-U_{0} B_{0}+B(t) U(t)\right]
$$

with (13) written as

$$
U(t)=U_{0}\left[1-\frac{B^{2}-\overline{B^{2}}}{2 M^{2} B_{0}^{2}}\right] .
$$

The result is that

$$
\begin{aligned}
\frac{B}{B_{0}}= & 1+\frac{A}{B_{0}} \cos \Omega t+\left(\frac{A}{B_{0}}\right)^{2}\left(\frac{1}{2 M^{2}}+\frac{\cos 2 \Omega t}{4\left(1-M^{2}\right)}\right) \\
& +\left(\frac{A}{B_{0}}\right)^{3} \frac{\left(4-M^{2}\right)}{64\left(1-M^{2}\right)^{2}}(\cos 3 \Omega t-\cos \Omega t)+O\left(\frac{A^{4}}{B_{0}{ }^{4}}\right)
\end{aligned}
$$

with

$\Omega^{2}=\Omega_{\mathrm{G}}{ }^{2}\left[\left(1-M^{2}\right)+\left(\frac{A}{B_{0}}\right)^{2} \frac{8-4 M^{2}-M^{4}}{8 M^{2}}+O\left(\frac{A^{4}}{B_{0}{ }^{4}}\right)\right]$.

It is of interest to contrast (90), which expresses the frequency in terms of $\Omega_{\mathrm{G}}$ and the $\mathrm{M}_{\mathrm{ACH}}$ number, with (86), which expresses it in terms of $\Omega_{\mathrm{G}}$ and the median dimensionless magnetic field. If one prefers to regard the known quantities as being $N_{0}, B_{0}, \Omega$, and $A$, one solves (90) by successive approximations for $M$ and then gets $U_{0}$ from (76).

It is important never to forget that $\tau$ and $t$ in (78) and (89) give the field as a function of time following the motion of a particle. Thus if we transform to a frame of reference with respect to which the wave has velocities $\bar{u}$ and $\bar{U}$ and the matter is stationary on the average, all other equations have their natural interpretation when applied to the running waves. But (78) and (89) do not give $b$ and $B$ as functions of time at a fixed point as the wave runs past; they give $b$ and $B$ at a particular particle which moves back and forth with the frequency of the wave.

If we neglect the terms in $a^{2}$ and $a^{3}$ in (78)(86) and those in $\left(A / B_{0}\right)^{2}$ and $\left(A / B_{0}\right)^{3}$ in (89) and (90), one gets the solution of the linearized equations for compressional magnetic waves in a plasma. This solution is well known, being a special case of more general solutions ${ }^{3,4,5}$ that are too numerous to list here. The expression for the phase velocity reduces in the linearized case to

$$
U_{0}=V_{\mathrm{A}} M=V_{\mathrm{A}}\left[1-\left(\Omega / \Omega_{\mathrm{G}}\right)^{2}\right]^{1 / 2} .
$$

Thus it is clear that all frequencies from 0 to $\Omega_{\mathrm{G}}$ are transmitted and that higher frequencies are not transmitted. (If the equations of motion are extend$e^{4,5}$ to include charge separation and the displacement current, a new mode will be introduced that propagates at higher frequency.) The possible range of velocities is from $V_{\mathrm{A}}$ to 0 and that of wavelength is from $\infty$ to 0 . All waves with $\Omega \ll \Omega_{\mathrm{G}}$ have essentially the same velocity and hence can be superposed to give a wave of arbitrary shape (provided no small wavelength, high $\Omega$, components are involved) that propagates with nearly unchanging form. This is the elementary compressional magneto-acoustic wave that propagates with the Alfvén velocity. The properties of this simple limiting form of the wave are easily derivable from the assumptions that the fluid moves only in the $x$-direction, that its conductivity is infinite, that there is a current density related to curl $\boldsymbol{B}$ and to the force on the fluid, and that elements of matter always remain on the same field line. If equations based on these principles are compared to those used above, it will be seen that it is only necessary to neglect $m_{p} w_{p}$ in (8); i.e. to neglect the inertia connected with changes in the current, to reduce our equations to the simpler case. And of course for very low frequency waves these inertial terms are negligible.

\section{Solitary waves}

In general the solutions found consist of infinite trains of waves of invariable form and frequency,

3 E. Åström, Ark. Fys. 2, 443 [1950].

${ }^{4}$ R. Lüst, Z. Astrophys. 37, 67 [1955].

5 G. H. A. Cole, Adv. Phys. 5, 452 [1956]. 
but there is one special case which while formally of the same character is essentially different. This is the solitary wave which is obtained when the frequency goes to zero. Under these circumstances there is only one crest, which travels as an isolated pulse. In the notation of Fig. 1, this solution is obtained when $\psi$ has its maximum value for a fixed $\varepsilon$, then $b_{3}$ and $b_{\mathrm{m}}$ coincide, and the representative point starts at the maximum of the $\varphi(b)$ curve. Since now two roots of $P(b)$ coincide, the elliptic integrals degenerate and it is possible to express the solutions in terms of more elementary functions. The analysis is also shortened by the fact that all averages, both those over time and those over space, are just the constant value at $\pm \infty$ of the quantity averaged. For the sections at $\pm \infty$ are infinitely long compared to the section near the crest where there are perceptible deviations from the constant value, and hence the latter have zero weight in computing the average. Thus $B_{0}$ and $N_{0}$ can now be regarded as the value of the corresponding quantities far from the wave crest and $U_{0}$ as the velocity of the wave. The dimensionless variables used above are no longer particular suitable since two different solitary waves in the same medium will, if they have different amplitudes and hence different velocities, have different values of $F$ and hence different scale factors for the conversion from $x$ to $\xi$ and from $t$ to $\tau$. Hence, although it seems unfortunate to complicate the discussion by defining yet another set of variables, it is easier to compare different solitary waves if the previous definitions are slightly modified by the insertion of factors which eliminate the dependence on $U_{0}$. To do this, note that at $x= \pm \infty$, $B=B_{0}, \quad b=b_{\mathrm{m}}, \quad U=U_{0}, \quad u=\bar{u}=1-b_{\mathrm{m}}{ }^{2}, \quad \varepsilon=\bar{b} u$ $=b_{\mathrm{m}} \bar{u}$. Hence by $(26), U_{0} F / \Pi=\bar{u}=1-b_{\mathrm{m}}{ }^{2}$. By (25) and (10),

$$
M^{2}=\frac{U_{0}{ }^{2} 4 \pi m_{\mathrm{t}} N_{0}}{B_{0}{ }^{2}}=\frac{U_{0} F}{2 \Pi b_{\mathrm{m}}{ }^{2}}=\frac{1-b_{\mathrm{m}}{ }^{2}}{2 b_{\mathrm{m}}{ }^{2}} .
$$

Therefore

$$
b_{\mathrm{m}}^{2}=1 /\left(2 M^{2}+1\right) \text {. }
$$

Next, use (74) to show that

$$
\Omega_{0}^{2}=\Omega_{\mathrm{G}}{ }^{2} \bar{u}^{2} / 2 \varepsilon^{2}=\Omega_{\mathrm{G}}{ }^{2}\left(2 M^{2}+1\right) / 2 .
$$

The new variables, defined both in terms of the usual quantities having dimensions and the dimensionless variables of section 4 , are

$$
\begin{aligned}
& X=\frac{\Omega_{\mathrm{G}}}{V_{\mathrm{A}}} x=\left[\frac{2 M^{2}+1}{2 M^{2}}\right]^{1 / 2} \xi, \\
& T=\Omega_{\mathrm{G}} t=\left[\frac{2}{2 M^{2}+1}\right]^{1 / 2} \tau,
\end{aligned}
$$

$$
\begin{gathered}
\beta=\frac{B}{B_{0}}-1=\frac{b}{b_{\mathrm{m}}}-1, \\
\mu=\frac{U}{V_{\mathrm{A}}}=\frac{2 M^{2}+1}{2 M} u, \\
Z=\frac{\Omega_{\mathrm{G}}}{V_{\mathrm{A}}} z_{\mathrm{r}}=\left[\frac{2 M^{2}+1}{2 M^{2}}\right] \zeta=\left[\left(\frac{m_{\mathrm{i}}}{m_{\mathrm{e}}}\right)^{1 / 2}+\left(\frac{m_{\mathrm{e}}}{m_{\mathrm{i}}}\right)^{1 / 2}\right] \frac{1}{M} \beta, \\
\vartheta_{\mathrm{s}}=\frac{e \Theta}{\left(m_{\mathrm{i}}-m_{\mathrm{e}}\right) V_{\mathrm{A}}{ }^{2}}=\left[\frac{2 M^{2}+1}{2 M}\right]^{2} \vartheta .
\end{gathered}
$$

The first equation of each definition could of course be used in general, but it is not convenient because the second equation does not hold except in the solitary wave case. The last equality of (99) holds by virtue of $(20)$, and hence with the aid of (18) we can regard curves of $\beta$ vs. $X$ as giving directly the shape of the trajectories.

To solve for the motion under these circumstances, we return to (45) and (50). Since $b_{3}=b_{\mathrm{m}}$ is now a double root whose value is specified in terms of $M^{2}$, we have

$$
\begin{aligned}
P(b) & =\left(b-b_{\mathrm{m}}^{2}\right)\left(-b^{2}+2 c_{1} b+c_{2}\right) \\
& =-b^{4}+2 b^{2}-4 \varepsilon b+(4 \psi-1)
\end{aligned}
$$

where $c_{1}$ and $c_{2}$ are constants whose values are to be determined by equating the coefficients of equal powers of $b$. Thus

$$
\begin{aligned}
P(b) & =\left(b-b_{\mathrm{m}}\right)^{2}\left(-b^{2}-2 b_{\mathrm{m}} b-3 b_{\mathrm{m}}^{2}+2\right) \\
& =4 b_{\mathrm{m}}{ }^{4} \beta^{2}\left(M^{2}-1-\beta-\frac{1}{4} \beta^{2}\right) .
\end{aligned}
$$

If this is substituted in (50) we get

$$
T= \pm \int \frac{\mathrm{d} \beta}{\beta\left(M^{2}-1-\beta-\frac{1}{4} \beta^{2}\right)^{1 / 2}}
$$

which integrates to

$$
\beta=\frac{2\left(M^{2}-1\right)}{1+M \cosh \left[\left(M^{2}-1\right)^{1 / 2} T\right]} .
$$

All constants of integration are chosen so that $X$, $T$, and $\vartheta_{\text {s }}$ are zero when $B$ is a maximum, and each \pm has the same sign as $X$. Next form

$$
\begin{aligned}
\mathrm{d} X & =\mu \mathrm{d} T=(1 / 2 M)\left[2 M^{2}+1-(\beta+1)^{2}\right] \mathrm{d} T \\
& =M \mathrm{~d} T \pm \frac{\mathrm{d}\left(M^{2}-1-\beta-\frac{1}{4} \beta^{2}\right)}{M\left(M^{2}-1-\beta-\frac{1}{4} \beta^{2}\right)^{1 / 2}} .
\end{aligned}
$$

Hence

$$
\begin{aligned}
X & =M T \pm(2 / M)\left[M^{2}-\left(1+\frac{1}{2} \beta\right)^{2}\right]^{1 / 2}, \\
\mu & =\left[\left(2 M^{2}+1\right)-(1+\beta)^{2}\right] / 2 M, \\
\vartheta_{\mathrm{s}} & =-\frac{1}{2}\left[\mu(T)^{2}-\mu(0)^{2}\right] .
\end{aligned}
$$

These expressions have been used to prepare the curves of Fig. 7 , which show $\beta, \mu$, and $\vartheta_{\mathrm{s}}$ as func- 


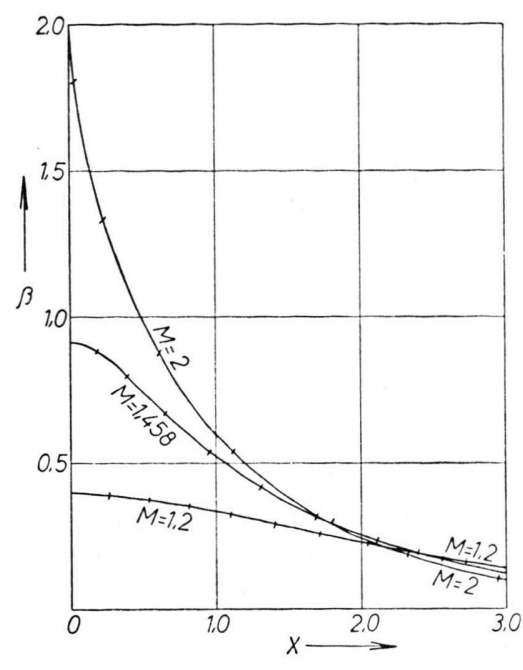

a

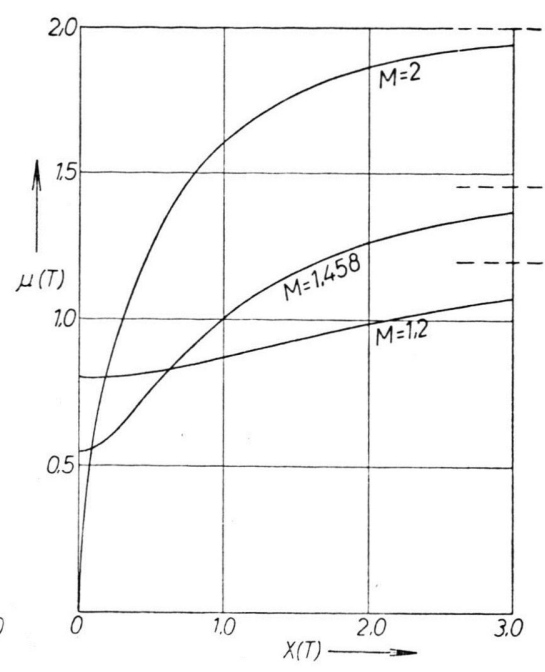

b

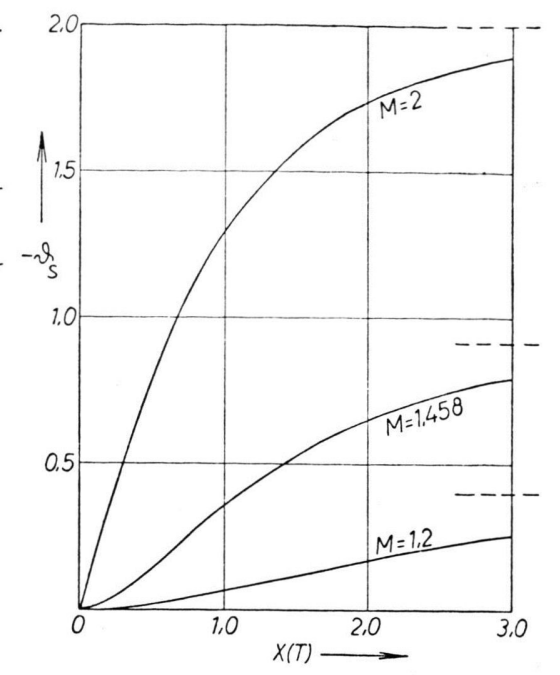

c

Fig. 7. Solitary waves in space for $M=2,1.458$ and 1.2. Half of the region near the crest is shown in each case as well as the asymptotic value at $\infty$. The other half of the wave is the mir ror image of the half shown. The marks on each curve show the points reached at equal intervals of dimensionless time, $\Delta T_{\mathrm{e}}=\frac{1}{3} .7$ a shows $\beta$ as a function of $X$ and hence shows both the variation of the magnetic field with position and the shape of the trajectories. $7 \mathrm{~b}$ and $7 \mathrm{c}$ show $\mu$ and $-\vartheta_{\mathrm{s}}$, respectively, as function of $X$. The values approached as $T \rightarrow \infty$ are indicated by dashed lines.

tions of $X$ for several values of $M$. Except for changes in scale, the middle curve of this figure is the same as that of Fig. 2. In order that the curves for $\beta$ in Fig. 7 represent the actual trajectories of electrons with the same scale in the horizontal and vertical directions, it is necessary, in the case of a plasma made of electrons and protons, that the curves be magnified in the vertical direction by factors of $10.7,14.7$, and 17.9 for $M=2,1.458$, and 1.2 , respectively. In each case the proton displacement is only $1 / 1836$ times as much.

For solitary waves, the minimum value of $M$ is 1 and the maximum value allowed by (40) is $M=2$, otherwise one gets looped trajectories and a more complicated analysis is required. It is of interest to note that the faster the solitary wave, i. e. the larger the value of $M$, the greater the maximum field and the sharper the crest. The time during which $\beta$ is more than half of its maximum value is very nearly $3 \sqrt{1-M^{2}} / \Omega_{\mathrm{G}}$; i. e., of the order of the geometric mean of the gyro-periods of the ions and electrons in the undisturbed magnetic field unless $M$ is very near to unity. The linear dimensions of the disturbance are of the general order of magnitude of $V_{\mathrm{A}} / \Omega_{\mathrm{G}}$; i. e. the geometric mean of the gyroradii of ions and electrons moving with the ALFvÉN velocity. Far from the crest the hyperbolic function in (103) may be approximated by an exponential and here the wave has the approximate shape

$$
\beta \doteq \frac{4\left(M^{2}-1\right)}{M} \exp \left[-\frac{\left(M^{2}-1\right)^{1 / 2} \Omega_{\mathrm{G}}|x|}{V_{\mathrm{A}} M}\right] \text {. }
$$

Thus for not too low densities the waves have scales large compared to the DeBye shielding length but small compared to the dimensions of the hydromagnetic waves usually considered.

\section{Hydromagnetic shock waves}

The original motivation for our treatment of hydromagnetic waves in a coordinate system moving with the waves was the hope that light would be cast on the structure of such waves in cases where it does not depend on the collisions between ions and electrons. If, for example, the solitary waves were not symmetrical but had allowed a waveless flow to enter on one side while waves emerged on the other, one would have a shock-like structure. Since (15) has the same form as the equation of motion of an undamped anharmonic oscillator, it is clear that all solution must be symmetrical about their maxima and minima and that the only solutions consistent with our assumptions are either isolated symmetrical solitary waves or wave trains of unvarying form. Thus the structure of a shock can be investigated only if our assumptions are modified. Of course only a very special problem was treated. 
Looped trajectories were excluded, charge separation and electrostatic plasma oscillations were excluded, thermal motions were excluded, collisions were excluded, and solutions of the non-linear wave equation that do not appear stationary in any coordinate system were excluded. However, if one examines the symmetry and the energy integrals of the equations of motion for looped trajectories, it can be proved that they have the same symmetry properties as the trajectories considered above. Hence the removal of this restriction can not give anything resembling shocks. If charge separation is allowed, a second class of waves characterized by electrostatic plasma frequencies will be introduced but the wave forms considered above will be only slightly modified, and it does not seem likely that they will change their symmetry properties, particularly in the linear approximation ${ }^{3,4}$. Treatment of the many complications introduced by thermal motions will have to be postponed to a later paper. If any collisions are allowed, solitary waves will no longer be possible and all waves will ultimately be damped to a smooth flow in a warm gas. This suggests a qualitative model of a shock in which a smooth flow of cold gas first tries to form a solitary wave. Even a few collisions between ions and electrons, with their oppositely directed $z$-motions, will mean that not all trajectories will be symmetrical and there will be a net displacement of charge. Thus $B$ will not fall quite to its original value and one will have generated a train of large amplitude, low frequency waves. At each succeeding crest there will be a few more collisions, the amplitude of the waves will fall, the frequency will increase, the temperature will rise, and in a distance of the order of the gas velocity times the mean time for a collision one will again have smooth flow of a warm gas. Thus the total shock will have a thickness related to the mean free path but it will have a detailed structure consisting of waves of the type we have been considering. However this model does not seem completely satisfactory in the limiting case in which the collision frequency becomes very small since the separation of the leading wave crests increases indefinitely. In fact, one seems forced to the conclusion that the transition region between flows of different properties, which must exist even in the limiting case where there are no thermal motions and no collisions, can not appear stationary in a system of coordinates that moves with the shock front. (We shall call this transition region a shock in a broad sense of the word.) Thus the exclusion of non-stationary solutions must be abandoned before shocks can be studied in this limiting case.

It is instructive to consider the formation of a shock from an ordinary longitudinal magneto-acoustic wave, not necessarily in a medium of zero temperature or with negligible collisions. When the wave length of such a wave is very long and the wave front has both a very small slope and a very small curvature, the wave propagates with a velocity which is the square root of the sum of the squares of the Alfvén and the sound velocities and with almost unchanging form. The only reason the form changes is because in the regions of excess density, which we may call crests (even though the wave is longitudinal), the field strength and temperature are both raised above the average value and hence both the Alfvén and sound velocities are increased. Thus the crests travel faster than the troughs and the parts of the wave where the density is increasing with time become steeper as the wave progresses and ultimately become shock fronts. If the wave is very long with both a trough and crest in which all conditions are constant over a long distance, then, in the frame of reference that moves with the transition region between, we have a flow of material of low density in on one side and of high density out on the other. And, as we saw, the transition region continuously shortens as the wave front steepens. Our basic problem is to discover what stops this shortening and leaves us with a shock front of finite rather than zero thickness. If we have no magnetic field, or if the field is small enough that the gyro-frequency is much less than the collision frequency, the answer is clear. One element of gas can act on another only through collisions and it takes a distance of the order of the mean free path for the low density, high velocity gas to be slowed down and compressed when it encounters the high density, low velocity gas. This process can be described either in terms of the deviations from the Maxwell-Boltzmann distribution over the thickness of the shock or approximately in terms of a viscosity and thermal conductivity. If the influence of collisions becomes small, the mean free path becomes long, and the thickness of the shock increases because the two kinds of gas interpenetrate for greater distances. However, if we consider a model in which there is a magnetic field but both collisions 
and thermal motion are negligible, we see that the situation is very different. In this case the temperature and sound velocity are zero everywhere and the wave propagates with the Alfvén velocity. The essential approximation implied by this statement is the neglect of inertial effects associated with changes in the current, as discussed at the end of section 7. The limit to the thinness of the shock front must be set by the point at which the inertial effects of the current carrying particles become significant and thus the thickness must certainly be greater than a flow velocity times a gyro- radius. Alternatively, one may say that the ions interact not by collisions but through the magnetic field and hence the maximum penetration of the high velocity stream into the low is of the order of the gyroradius. Thus as collisions become negligible and the collision frequency becomes less than the gyrofrequency, the penetration does not grow with the mean free path but is limited by the gyro-radius. Nevertheless, the analysis of the previous sections shows that when there are no collisions the shock can not be described by a stationary flow. Thus the region between the smooth flow must now be occupied by some kind of wave train whose length continually grows as the ends of the wave train eat into the regions of smooth flow. Ultimately this length becomes so long that collisions can not be neglected and they damp out the waves at the downstream end, leaving high density and temperature there. Thus the total length of the transition region is determined by the mean free path but the scale of the detailed structure is the gyro-radius. With this model, there seems to be no limit to the increase in the magnetic field strength between the trough and the part of the wave train where collisions have not yet increased the temperature. Once the temperature rises, expansion will limit the maximum field strength to the well known value of four times the minimum field.

It is possible to find an analogue of the RankineHugeniot conditions for a hypothetical model of hydrodynamic shocks in which the regions on the two sides of the "shock front" are occupied by cold plasmas of different mean densities and magnetic field strengths, in each region there being suitable wave trains of the type considered above. Each train will appear stationary in its own co-moving coordinate system, but each will appear to be a running wave in the other waves co-moving system and in the system that moves with the shock front. The desired conditions are found when one requires that the time averages of the various fluxes are conserved across the shock front. This model differs from that ordinarely treated in that: 1 . The gas is cold on both sides of the shock front, there being no generation of entropy. 2. There turns out to be a one parameter family of possible flows on the down stream side instead of only two possible flows. 3 . The transition region between the two sides of the shock is in a fluctuating state so that one has conservation only on the average over time. In an ordinary shock front it is assumed that even in the transition region the model is in a steady state and hence that the fluxes (including in their expression the terms that allow for the dissipative phenomena) are the same everywhere and at all times. This suggests that in our case it would be more realistic to regard the fluctuating transition region not as producing periodic waves but as generating a statistically fluctuating wave train on the down stream side which did not appear stationary in any coordinate system but which had the proper average fluxes. Since such wave trains have not been investigated, we shall not consider them further.

We shall confine our attention to wave trains which, in a suitable frame of reference, appear stationary, but shall treat them also in moving frames where they form running waves. It is therefore necessary to see how the various fluxes transform under GaLILEAN transformations. This is easily done if we express them in terms of the density characterization since we know how densities transform. It is convenient to designate the fluxes in the frame of reference in which the waves appear stationary as eigen-fluxes and to attach an ${ }^{*}$ to fluxes in all other frames; this is consistent with the fact that until now all fluxes considered have been eigen-fluxes. Let $V$ be the phase velocity of the wave train in the *system. It is then the velocity of the eigen-system with respect to the * system, and

$$
U^{*}=U+V
$$

where $U^{*}$ and $U$ are the $x$-components of the material velocity in the ${ }^{*}$ and eigen-systems, respectively, Since the particle density, $N$, is the same in both frames of reference and since $F=m_{\mathrm{t}} N U$ and $F^{*}=m_{\mathrm{t}} N U^{*}$, we get

$$
F^{*}=F+V m_{\mathrm{t}} N
$$

as an expression for the variable mass flux density in an arbitrary system in terms of the variable mass density and the constant eigen-flux and phase velocity. Since $B$ has the same value in either frame (here and in the future we neglect without explicit mention terms of order $V^{2} / c^{2}$ ), the LoRentz transformation gives us

$$
c E^{*}=c E+V B \text {. }
$$


Since the momentum fluxes are $\Pi=\left(B^{2} / 8 \pi\right)+m_{\mathrm{t}} N U^{2}$, $\Pi^{*}=\left(B^{2} / 8 \pi\right)+m_{\mathrm{t}} N U^{* 2}$, we get

$$
\Pi^{*}=\Pi+2 V F+V^{2} m_{\mathrm{t}} N .
$$

Let us postpone consideration of the more difficult transformation law for energy flux density.

We now use these expressions to get the various fluxes on the two sides of a shock front in the frame of reference in which the shock front is at rest. Since we allow the transition region to store mass, momentum, energy, and magnetic field flux over the period of the waves but require that on the average it not serve as a source or sink of these quantities, then the average value of each flux must not change across the shock. The time averages of $N$ and $B$ at a fixed point are the same as the space averages over a wave of unchanging form and velocity, but are not the same as the time averages following the motion of a single particle, which was denoted by a bar in section 6. It is convenient to express $\langle N\rangle$ and $\langle B\rangle$ in terms of $\bar{U}$ by means of (68) and (69) where $\bar{U}$ is the mean velocity in the eigensystem. Thus if $\Delta Q$ denotes the difference in the values of any quantity, $Q$, on the two sides of the shock, we must have

$$
\begin{aligned}
& \Delta\left[F\left(1+V \bar{U}^{-1}\right)\right]=0, \\
& \Delta\left[c E\left(1+V \bar{U}^{-1}\right)\right]=0, \\
& \Delta\left[\Pi+V F\left(2+V \bar{U}^{-1}\right)\right]=0 .
\end{aligned}
$$

It is perhaps not obvious that (113) is correct since $c E^{*}$ is not obviously the flux of magnetic lines of force. However, this interpretation follows directly from

$$
\frac{\partial B}{\partial t}+\frac{\partial\left(c E^{*}\right)}{\partial x}=0
$$

which is one of Maxwell's equations and has the same form as the expression connecting mass density and mass flux. In case (113) still does not seem obvious, one integrates $c E^{*} \mathrm{~d} l$ around a rectangular path two of whose sides are along $e_{z}$ on opposite sides of the shock front. One uses curl $c E^{*}=-\dot{B}$ and the condition that the magnetic flux through the loop has a constant average value to get $\Delta\left\langle c E^{*}\right\rangle=0$.

The eigen-flux $\Psi$ can be expressed by (24) in terms of $N, U, m_{\mathrm{r}} w_{\mathrm{r}}^{2}, B$, and $E$; and hence $m_{\mathrm{r}} w_{\mathrm{r}}{ }^{2}$ can be expressed in terms of $\Psi$ and the other quantities. The corresponding flux $\Psi^{*}$ in any other frame of reference is obtained from the expression for $\Psi$ by replacing $U$ by $U^{*}, c E$ by $c E^{*}$, and $m_{\mathrm{r}} w_{\mathrm{r}}{ }^{2}$ by the expression in terms of $\Psi$. This last step was not be necessary for the other fluxes and implies that the expression for $\Psi^{*}$ depends on more than $V$ and the fluxes and densities. One gets, using (108), (110), and the formulas for the other eigen-fluxes,

$$
\begin{gathered}
\Psi^{*}=\frac{1}{2} m_{\mathrm{t}} N U^{* 3}+\frac{1}{2} m_{\mathrm{r}} N U^{*} w_{\mathrm{r}}^{2}+\left(B c E^{*} / 4 \pi\right) \\
=\Psi\left(1+V U^{-1}\right)+V \Pi+\frac{3}{2} V^{2} F+\frac{1}{2} V^{3} m_{\mathrm{t}} N \\
+\left(V B^{2} / 8 \pi\right)-(V B c E / 4 \pi U) .
\end{gathered}
$$

In order to average this over space, the variable terms may be converted to reduced variables by $(25)-(29)$ and averaged by the use of (58), (36), and (59). Converting back to dimensional variables one gets

$\left\langle\Psi^{*}\right\rangle=\Psi\left(1+\frac{1}{3} V U^{-1}\right)+\frac{4}{3} V \Pi$

$$
+\frac{3}{2} V^{2} F+\frac{1}{2} V^{3} m_{\mathrm{t}}\langle N\rangle .
$$

Thus conservation of energy across the front requires that we add to (112), (113), and (114) the fourth condition

$$
\begin{aligned}
\Delta\left[\Psi\left(1+\frac{1}{3} V \bar{U}^{-1}\right)+\frac{4}{3} V \Pi\right. & \text { (118) } \\
\left.+\frac{1}{2} V^{2} F\left(3+V \bar{U}^{-1}\right)\right] & =0 .
\end{aligned}
$$

The case of smooth, waveless flow is anomalous in that when there are no waves there is nothing to set one frame apart from the others. Thus all sets of fluxes consistent with smooth flow may be regarded as eigenfluxes and (109), (110), (111), and (117) may be regarded as formulas for converting the eigen-fluxes in any frame of reference into those in a system having velocity $V$. For smooth flow $\left\langle\Psi^{*}\right\rangle=\Psi^{*}$ and $\bar{U}=U$. If one wishes to apply (112), (113), (114), and (115) to a situation where there is smooth flow on one side of the front, it is only necessary to set $V=0$ on that side of the front, using a frame of reference moving with the shock for the eigen-fluxes. Since with smooth flow $w_{\mathrm{r}}=0$ and $c E=B U$, one can solve the expressions given above for $F, \Pi$, and $\Psi$ in terms of $m_{\mathrm{t}}, N$, $U, B$, and $w_{\mathrm{r}}$ to get $U$ in terms of the fluxes.

$$
U=\frac{12(c E)^{2} \Pi+32 \pi \Psi^{2}}{16 \pi \Pi \Psi+9 F(c E)^{2}} .
$$

This can be given other forms because there is an identity, implied by (125) and (126) below, connecting the fluxes that must be fulfilled if there is to be smooth flow.

In these equations there appear six quantities to be evaluated on each side of the shock but only five are independent because $U$ is determined by the eigenfluxes and may regarded as expressed in terms of them by complicated elliptic functions. One can also use Fig. 4 or eq. (81) to approximate the functional dependence. For smooth flow one can use (119). In practice it will probably be best to characterize the regions by the value of $V, \tilde{a} \mathfrak{b}, F$, and $\Pi$. If the five quantities that determine the conditions on one side of the shock front are fixed, we have equations that restrict the quantities on the other side and thus there remains in general a one parameter family of solutions.

To show that real, physically reasonable solutions do exist for which conditions are different across the shock, we shall exhibit such a solution. To get it, we apply (112), (113), (114), and (118) to the case in which there is a running wave characterized by eigenfluxes $F_{\mathrm{w}}, c E_{\mathrm{w}}, \Pi_{\mathrm{w}}, \Psi_{\mathrm{w}}$, and $V_{\mathrm{w}}$ on one side of the front and a smooth flow characterized by the corres- 
ponding quantities with subscripts $\mathrm{s}, V_{\mathrm{s}}=0$, on the other. Regard $V_{\mathrm{s}}=0, F_{\mathrm{w}}, c E_{\mathrm{w}}, \Pi_{\mathrm{w}}$, and $\Psi_{\mathrm{w}}$ as given. Use the four continuity conditions and the identity that the fluxes must satisfy for a smooth flow to determine the remaining quantities. In order to carry out such a solution it is desirable to define

$$
\beta=F_{\mathrm{w}} / F_{\mathrm{s}}, \quad \gamma=\Pi_{\mathrm{w}} / \Pi_{\mathrm{s}}
$$

and to reduce the equations to dimensionless form by $(25)-(29)$, getting

$$
\begin{aligned}
V_{\mathrm{w}} & =\beta(1-\gamma) \Pi_{\mathrm{w}} / \gamma(\beta+1) F_{\mathrm{w}}, \\
\bar{u}_{\mathrm{w}} & =\beta^{2}(\gamma-1) / \gamma\left(\beta^{2}-1\right) \\
\gamma & =\left[\left(1-\bar{u}_{\mathrm{w}}\right)+\left(\bar{u}_{\mathrm{w}} / \beta^{2}\right)\right]^{-1}, \\
\varepsilon_{\mathrm{s}} & =\varepsilon_{\mathrm{w}} \gamma^{3 / 2} / \beta^{2}, \\
\psi_{\mathrm{s}}=\psi_{\mathrm{w}} \frac{(2 \beta+1)}{3 \beta^{2}} & \gamma^{2}
\end{aligned}
$$

These may appear somewhat formidable for convenient simultaneous solution, but if one characterizes a particular wave by its values of $F_{\mathrm{w}}, \Pi_{\mathrm{w}}, \mathfrak{b}$, and $\tilde{a}$, one can get the corresponding values of $\varepsilon_{\mathrm{w}}, \psi_{\mathrm{w}}$, and $\bar{u}_{\mathrm{w}}$ from Fig. 3 and 4 , or more accurately from (46), $(47)$, and the results of a numerical integration.

Then by successive trials, find a value of $\beta$ which gives values of $\gamma, V_{\mathrm{w}}, \varepsilon_{\mathrm{s}}$, and $\psi_{\mathrm{s}}$, for which the last two quantities satisfy the parametric equations for smooth flow,

$$
\begin{aligned}
\varepsilon_{\mathrm{s}} & =2 M_{\mathrm{s}}^{2} /\left(2 M_{\mathrm{s}}^{2}+1\right)^{3 / 2}, \\
\psi_{\mathrm{s}} & =M_{\mathrm{s}}^{2}\left(M_{\mathrm{s}}^{2}+2\right) /\left(2 M_{\mathrm{s}}^{2}+1\right)^{2},
\end{aligned}
$$

where $M_{\mathrm{S}}$ is the $\mathrm{M}_{\mathrm{ACH}}$ number of the smooth flow with respect to the shock front and these formulas are easily derived from (68) - (71).

As a specific example, this process was applied to the wave for which $a=0.2, \mathfrak{b}=0.7$, and hence $\bar{u}_{\mathrm{w}}=0.5320, \varepsilon_{\mathrm{W}}=0.3289$, and $\psi_{\mathrm{w}}=0.3051$. The process of solution outlined above gives $\beta=0.9484, \gamma=$ 0.9439 , and $M_{\mathrm{s}}=1.627$. If $V_{\mathrm{As}}$ and $V_{\mathrm{Aw}}$ denote the Alfvén velocities in the two regions, one finds that the phase velocity of the wave system refered to the shock is

$$
V_{\mathrm{w}}=0.0557 V_{\mathrm{As}}
$$

and that the relations between the states on opposite sides of the transition region are given by

$$
\begin{gathered}
\left\langle B_{\mathrm{w}}\right\rangle=1.507 B_{\mathrm{s}}, \quad\left\langle N_{\mathrm{w}}\right\rangle=1.507 N_{\mathrm{s}}, \\
M_{\mathrm{s}}=U_{\mathrm{s}} / V_{\mathrm{As}}=1.627, \quad M_{\mathrm{w}}=U_{\mathrm{ws}} / V_{\mathrm{Aw}}=0.717 .
\end{gathered}
$$

Here $\bar{U}_{\mathrm{ws}}$ is the mean velocity in the wave region but in the frame of reference in which the shock is stationary

$$
\begin{aligned}
\bar{U}_{\mathrm{w}} & =1.024 V_{\mathrm{As}}, & \bar{U}_{\mathrm{Ws}} & =1.080 V_{\mathrm{As}}=U_{\mathrm{w}}+V_{\mathrm{w}}, \\
c E_{\mathrm{s}} & =1.627 B_{\mathrm{s}} V_{\mathrm{s}}, & c E_{\mathrm{w}} & =1.543 B_{\mathrm{s}} V_{\mathrm{As}}, \\
\Pi_{\mathrm{s}} & =3.15 B_{\mathrm{s}}^{2} / 4 \pi, & \Pi_{\mathrm{w}} & =2.97 B_{\mathrm{s}}{ }^{2} / 4 \pi, \\
\Psi_{\mathrm{s}} & =3.78 V_{\mathrm{As}} B_{\mathrm{s}}{ }^{2} / 4 \pi, & \Psi_{\mathrm{w}} & =3.40 V_{\mathrm{As}} B_{\mathrm{s}}^{2} / 4 \pi .
\end{aligned}
$$

The magnetic field in the waves varies between $1.22 B$ and $2.19 B_{\mathrm{s}}$.

It may be of interest to contrast the above conservation conditions across a shock when on has waves in material whose temperature is zero with the corresponding conditions when one has no waves and no collisions but an unspecified mechanism that produces entropy and gives an increase in temperature across the shock. If one has no collisions and the direction of propagation of the shock is normal to the magnetic field, the motions of the particles parallel to the field is unaffected by the shock and the heating corresponds to an increase of the energy of motion normal to the field and is associated with an increase in the corresponding terms of the pressure tensor. If the particle velocities are assumed to be isotropic in the plane normal to the magnetic field, then we may disregard the coordinate parallel to the field and regard the gas as a two dimensional isotropic gas with pressure, $p_{Q}$, and internal energy per unit mass, $u_{Q}$ (although the subscript $\mathrm{Q}$ is not needed here to distinguish these quantities from the corresponding quantities, $p_{\mathrm{P}}$ and $u_{\mathrm{P}}$, associated with motion parallel to the field, it is needed to avoid confusion with the previous usage of $u$ for a dimensionless velocity). The connection between presssure and internal energy for an ideal two dimension ionic gas is

$$
p_{Q}=\varrho u_{Q}=\frac{1}{2} \varrho i_{Q}
$$

where $\varrho=m_{\mathrm{t}} N$ is the density and $i_{Q}$ is the specific enthalpy associated with the transverse motion. Then the facts that curl $E=0$ in a system in which the shock appears stationary and that we have conservation of mass, momentum, and energy give, in the usual way ${ }^{6}$, the conditions

$$
\begin{array}{cr}
U_{\mathrm{I}} B_{\mathrm{I}}=U_{\mathrm{II}} B_{\mathrm{II}}, & (128) \\
\varrho_{\mathrm{I}} U_{\mathrm{I}}=\varrho_{\mathrm{II}} U_{\mathrm{II}}, & (129) \\
\varrho_{\mathrm{I}} U_{\mathrm{I}}^{2}+p_{\mathrm{QI}}+\left(B_{\mathrm{I}}^{2} / 8 \pi\right)=\varrho_{\mathrm{II}} U_{\mathrm{II}}+p_{\mathrm{QII}}+ & \left(B_{\mathrm{II}}^{2} / 8 \pi\right), \\
U_{\mathrm{I}}\left[\frac{1}{2} \varrho_{\mathrm{I}} U_{\mathrm{I}}^{2}+\varrho_{\mathrm{I}} i_{\mathrm{QI}}+\left(B_{\mathrm{I}}^{2} / 4 \pi\right)\right] & (130) \\
=U_{\mathrm{II}}\left[\frac{1}{2} \varrho_{\mathrm{II}} U_{\mathrm{II}}^{2}+\varrho_{\mathrm{II}} i_{\mathrm{QII}}+\left(B_{\mathrm{II}}^{2} / 4 \pi\right)\right] .
\end{array}
$$

It will be noted that while the connection of (129) and (128) to (112) and (113) is reasonably close, it is not easy to see how the remaining equations can be interpreted as equivalent. This is unfortunate as $(128)-(131)$ are relatively easy to solve

${ }^{6}$ R. Lüst, Z. Naturforschg. 8 a, 278 [1953]; 10 a, 125 [1955]. 
for conditions on one side of a shock given those on the other. To do this introduce the energy density ratio, $R_{i}$, the $\mathrm{MACH}_{\mathrm{ACH}}$ number, $M_{i}, i=\mathrm{I}$ or II, and $C$, the compression ratio across the shock, by

$$
\begin{array}{r}
R_{i}=\left(\varrho_{i} u_{i}+\frac{B_{i}{ }^{2}}{8 \pi}\right) /\left(\frac{B_{i}{ }^{2}}{8 \pi}\right)=\frac{p_{i}^{*}}{p_{\mathrm{M} i}}, \quad \\
\varrho_{i} U_{i}{ }^{2}=\varrho_{i} M_{i}{ }^{2}\left(2 p_{\mathrm{Q} i}+\frac{B_{i}{ }^{2}}{4 \pi \varrho_{i}}\right)=M_{i}{ }^{2} R_{i} \frac{B_{i}{ }^{2}}{4 \pi},(133) \\
\varrho=B_{\mathrm{II}} / B_{\mathrm{I}}=\varrho_{\mathrm{II}} / \varrho_{\mathrm{I}}=U_{\mathrm{I}} / U_{\mathrm{II}}=\left(R_{\mathrm{I}} M_{\mathrm{I}}{ }^{2} / R_{\mathrm{II}} M_{\mathrm{II}}{ }^{2}\right)^{1 / 3}
\end{array}
$$

where $p_{M i}$ is the magnetic pressure $\left(B_{i}{ }^{2} / 8 \pi\right), p_{i}{ }^{*}$ is the total pressure $\left(p_{Q i}+p_{M i}\right)$, the velocity of sound is the quantity in parentheses in (133), and the last equality of (134) follows because

$$
R_{i} M_{i}{ }^{2}=4 \pi \varrho_{i} U_{i}^{2} / B_{i}{ }^{2} .
$$

Equations (134) ensure that (128) and (129) are satisfied, and by using (127), we can express (130) and (131) in terms of the new variables. The result is that either $C=1$ and there is no change across the shock or

$$
\begin{gathered}
R_{\mathrm{II}}=R_{\mathrm{I}} \frac{(3 C-1)}{C^{2}(3-C)}, \\
M_{\mathrm{I}}^{2}=2 C /(3-C) ; \quad C=3 M_{\mathrm{I}}^{2} /\left(2+M_{\mathrm{I}}^{2}\right) .
\end{gathered}
$$

Thus if one is given $B_{\mathrm{I}}, \varrho_{\mathrm{I}}, u_{\mathrm{QI}}$ or $p_{\mathrm{QI}}$, and $U_{\mathrm{I}}$; one evaluates $R_{\mathrm{I}}$ and $M_{\mathrm{I}}$, uses (136) to find $C$, and then easily finds all quantities in region II. If one is given $C$ instead of $U_{\mathrm{I}}$, one finds $M_{\mathrm{I}}^{2}$ and then $U_{\mathrm{I}}$ from (133). These equations are, of course, not new but are the forms obtained when one puts $\gamma=2$ in the formulas of Lüst ${ }^{6}$. In material flows across the shock from region I to region II, the inequalities

$$
1 \leqq C \leqq 3, \quad 1 \leqq R_{\mathrm{II}} / R_{\mathrm{I}}<\infty
$$

are implied by the requirements that the entropy increase across the shock and that $R$ be positive since $\varrho, u_{Q}$, and $B^{2}$ are positive. It will be recalled that when some energy can be transfered to particle motion along the magnetic field, there can be more compression before the pressures balance the momentum flux and the maximum compression ratio is 4 . If energy can be transfered to still other forms, such as dissociation of ions or the production of waves of the type considered above, then still higher values of $C$ should be possible.

7 W. Marshall, Proc. Roy. Soc., Lond. A 233, 367 [1955].

8 H. K. Sen, Phys. Rev. 102, 5 [1956].

9 M. H. Rose, On the Structure of a Steady Hydromagnetic Shock, New York University 1956.
If these equations are used to compute the downstream conditions for a shock having the same upstream conditions as those in the example following (126), i. e. $M_{\mathrm{s}}=1.627$, one gets $C=1.71$ and hence

$B_{\mathrm{II}}=1.71 B_{\mathrm{S}}, \varrho_{\mathrm{I}} u_{\mathrm{QI}}=0$,

$$
\varrho_{\mathrm{II}} u_{\mathrm{QII}}=p_{\mathrm{QII}}=0.27 B_{\mathrm{S}}{ }^{2} / 8 \pi .
$$

Thus this magnetic field is very nearly the median of the values for the wave and is higher than the mean field in the wave but, of course, lower than the maximum, which may always be expected to overshoot the value given by (128).

In conclusion, let us briefly note the connection of this investigation with earlier work on the structure of hydromagnetic shocks. Marshall ${ }^{7}, \mathrm{SEN}^{8}$, and $\operatorname{Rose}^{9}$ have all neglected the inertia associated with the current carrying elements and hence smooth over the wave structure we find. This neglect of inertia is equivalent to setting $\ddot{B}=0$ in (15). This Sen does; Marshall and Rose replace this term by one proportional to $1 / \sigma \cdot \mathrm{d} B / \mathrm{d} x$, where $\sigma$ is the electrical conductivity. This replaces the inertial term by a frictional one and allows non-periodic solutions. Since a non-zero value of $1 / \sigma$ implies collisions, this should lead to a shock thickness that depends on the mean free path that determines the conductivity. Marshall and Sen consider in addition the effects of viscosity and thermal conductivity, which, as expected, leads to a shock thickness of the order of the mean free path. Rose neglects these terms on the assumption that the mean free path is very large. Rose ${ }^{9}$ and Rose and GRAD ${ }^{10}$ conclude that the complete shock thickness is of the order of the gyro-radius, a result that seems surprising on the basis of the above analysis. It appears that the difference may arise partly because of Rose's basic inconsistency in ignoring collisions in part of his analysis but including them when he uses an electrical conductivity, partly because the proportionality factors he discards are not of order unity, and partly because at a shock the mean free path is determined not by the thermal velocity but by the flow velocity which Rose takes to be proportional to $B$ in the region under discussion. In all

10 M. H. Rose and H. Grad, Bull. Amer. Phys. Soc. II, 1, 18 [1956]. 
three treatments the pressure is taken to be the same along and normal to the magnetic field as is appropriate where collisions maintain an isotropic velocity distribution. Thus it will be seen that these treatments are appropriate for the considerations of the effects of collisions in producing the relatively gradual transition from one side of the complete shock to the other, while our treatment is better adapted to the study of the detailed structures. Of course, our treatment is relevant only in the limited range of densities, field strengths, and temperatures for which the gyro-frequency is much greater than the collision frequency and the pressure is negligible compared to $\Pi$.

We are greatly indebted to Professor L. Biermann for pointing out the importance of this problem and for his most helpful suggestions. We wish to thank Mr. Katterbach for plotting the figures and Mrs. G. HaIN for coding and carrying out the computations on the $\mathrm{G} 2$.

One of us (L. D.) is indebted to the U.S. National Science Foundation for a Fellowship and to the Fulbright Commission for a Travel Grant that made it possible for him to take part in this research. A. S. is indebted to Dr. H. PEтscher of AVCO Research Laboratories for many discussions on this subject.

\title{
Zur Stabilität zylindersymmetrischer Plasmakonfigurationen mit Volumenströmen
}

\author{
Von K. HaIN und R. Lüst \\ Aus dem Max-Planck-Institut für Physik, Göttingen \\ (Z. Naturforschg. 13 a, 936-940 [1958] ; eingegangen am 31. Juli 1958)
}

\begin{abstract}
The stability of cylindersymmetric plasma configuration with volume currents is investigated by the method of small perturbations. The problem is reduced to only one eigen-value differential equation of second order. For a special current distribution with relativly strong concentration to the axis the eigen-values are computed numerically. For this current distribution specially for long wave lengths instability shows up. The rates of growth for different kinds of pertubations are given as a function of the wave length.
\end{abstract}

In einer vorangegangenen Untersuchung von HAIN, Lüst und SCHLÜTER ${ }^{1}$ waren allgemeine Bedingungen für die Stabilität hydromagnetischer Gleichgewichtskonfigurationen untersucht worden. In weitgehend analoger Form sind ähnliche Fragen auch in Princeton von Bernstein, Frieman, Kruskal und Kulsrud ${ }^{2}$ behandelt worden. Nach KRUskal und Schwarzschild $^{3}$ ist es bekannt, daß eine zylindersymmetrische Gleichgewichtskonfiguration mit nur an der Oberfläche befindlichen Strömen die sog. Pinch-Instabilität aufweist. Von Rosenbluth ${ }^{4}$ und TAyler $^{5}$ wurde nun gezeigt, daß eine solche Pinchentladung durch besondere Magnetfelder und Außenleiter zu stabilisieren ist.

In allen praktischen Fällen werden die elektrischen Ströme wegen der endlichen elektrischen Leitfähigkeit nicht ausschließlich an der Oberfläche fließen, sondern die Ströme werden im Laufe der Zeit in das

1 K. Hain, R. Lüst u. A. Schlüter, Z. Naturforschg. 12 a, 833 [1957]; im folgenden als I zitiert.

2 I. B. Bernstein, E. A. Frieman, M. P. Kruskal u. R. M. KulsRud, Proc. Roy. Soc., Lond. A 244, 17 [1958].
Plasma eindringen. Ein möglicher Grenzfall wäre dann z. B., daß die sich entwickelnden achsenparallelen Volumenströme konstant sind, d. h. nicht mehr vom Abstand von der Symmetrieachse abhängen. In diesem Fall ergeben die Rechnungen von TAYLER, $\mathrm{da} ß$ dann auch bei Gegenwart von äußeren Magnetfeldern und Außenleitern eine Stabilisierung nicht $\mathrm{zu}$ erreichen ist. Von besonderem Interesse ist es deshalb, wie weit die Volumenströme in das Plasma eindringen dürfen, bis die Gleichgewichtskonfiguration instabil wird.

Im folgenden soll nun der Einfluß von Volumenströmen auf die Stabilität eines Plasmazylinders genauer untersucht werden. In I war die Stabilität von Gleichgewichtskonfigurationen mit Hilfe der Methode der kleinen Störungen behandelt worden; dabei zeigte sich, daß das Stabilitätsverhalten durch die folgende Vektordifferentialgleichung 2. Ordnung in

3 M. P. Kruskal u. M. Schwarzschild, Proc. Roy. Soc., Lond. A 223, 348 [1954].

4 M. Rosenbluth, Los Alamos Sci. Lab. LA-2030.

5 R. J. TAyler, Proc. of the third Int. Conf. on Ionized Gases, p. 903 [1957]; Proc. Phys. Soc. B 70, 1049 [1957]. 